\title{
VISCOELASTICITY WITH TIME-DEPENDENT MEMORY KERNELS. PART II: ASYMPTOTIC BEHAVIOR OF SOLUTIONS
}

\author{
MONICA CONTI, VALERIA DANESE AND VITTORINO PATA
}

\begin{abstract}
We continue the analysis on the model equation arising in the theory of viscoelasticity

$$
\partial_{t t} u(t)-\left[1+k_{t}(0)\right] \Delta u(t)-\int_{0}^{\infty} k_{t}^{\prime}(s) \Delta u(t-s) \mathrm{d} s+f(u(t))=g
$$

in the presence of a (convex, nonnegative and summable) memory kernel $k_{t}(\cdot)$ explicitly depending on time. Such a model is apt to describe, for instance, the dynamics of aging viscoelastic materials. The earlier paper [4] was concerned with the correct mathematical setting of the problem, and provided a well-posedness result within the novel theory of dynamical systems acting on time-dependent spaces, recently established by Di Plinio et al. [14]. In this second work, we focus on the asymptotic properties of the solutions, proving the existence and the regularity of the time-dependent global attractor for the dynamical process generated by the equation. In addition, when $k_{t}$ approaches a multiple $m \delta_{0}$ of the Dirac mass at zero as $t \rightarrow \infty$, we show that the asymptotic dynamics of our problem is close to the one of its formal limit
\end{abstract}

$$
\partial_{t t} u(t)-\Delta u(t)-m \Delta \partial_{t} u(t)+f(u(t))=g
$$

describing viscoelastic solids of Kelvin-Voigt type.

\section{INTRODUCTION}

Given a bounded domain $\Omega \subset \mathbb{R}^{3}$ with smooth boundary $\partial \Omega$, take the Hilbert space $\mathrm{H}=L^{2}(\Omega)$, and let

$$
A=-\Delta \quad \text { with domain } \mathfrak{D}(A)=H^{2}(\Omega) \cap H_{0}^{1}(\Omega)
$$

be the Laplace-Dirichlet operator on $H$. In the earlier work [4], for any initial time $\tau \in \mathbb{R}$, we considered the evolution problem in the unknown variable $u=u(t):[\tau, \infty) \rightarrow \mathrm{H}$

$$
\begin{gathered}
\partial_{t t} u(t)+A u(t)+\int_{0}^{\infty} \mu_{t}(s) A \eta^{t}(s) \mathrm{d} s+f(u(t))=g, \\
\eta^{t}(s)= \begin{cases}u(t)-u(t-s), & 0<s \leq t-\tau, \\
\eta_{\tau}(s-t+\tau)+u(t)-u_{\tau}, & s>t-\tau,\end{cases} \\
\left\{\begin{array}{l}
u(\tau)=u_{\tau}, \\
\partial_{t} u(\tau)=v_{\tau}, \\
\eta^{\tau}(s)=\eta_{\tau}(s), \quad s>0,
\end{array}\right.
\end{gathered}
$$

2000 Mathematics Subject Classification. 35B41, 45K05, 73E50, 74D99.

Key words and phrases. Viscoelasticity, Kelvin-Voigt model, memory, time-dependent kernels, processes on time-dependent spaces, time-dependent global attractors. 
where the initial values $u_{\tau}, v_{\tau}$ and $\eta_{\tau}=\eta_{\tau}(s)$ are assigned data. Here, $f(u)$ is a nonlinear term, $g$ a constant-in-time external force, while the function $\mu_{t}=\mu_{t}(s)$ of the variable $s>0$ is the so-called memory kernel, which is allowed to exhibit an explicit dependence on time, and is supposed to be absolutely continuous on $\mathbb{R}^{+}=(0, \infty)$, nonincreasing and summable (hence nonnegative) for every fixed $t$. As discussed in detail in [4], problem (1.1)-(1.3) arises in the theory of uniaxial deformations in isothermal viscoelasticity (see e.g. $[3,18,30])$. In this context, the auxiliary variable ${ }^{1} \eta=\eta^{t}(s)$ contains all the information on the past history (i.e. for times $t<\tau$ ) of the axial displacement field $u$. Indeed, assuming $u$ to be known for all past times, and interpreting the initial value $\eta_{\tau}$ as

$$
\eta_{\tau}(s)=u(\tau)-u(\tau-s)
$$

then (1.1)-(1.2) take the more familiar form ${ }^{2}$

$$
\partial_{t t} u(t)+\left[1+k_{t}(0)\right] A u(t)+\int_{0}^{\infty} k_{t}^{\prime}(s) A u(t-s) \mathrm{d} s+f(u(t))=g,
$$

where the (nonnegative) convex function

$$
k_{t}(s)=\int_{s}^{\infty} \mu_{t}(y) \mathrm{d} y
$$

is the integrated memory kernel, supposed to be summable as well, which satisfies by construction the relation

$$
k_{t}^{\prime}(s)=-\mu_{t}(s) .
$$

Since a formal integration by parts yields

$$
\int_{0}^{\infty} k_{t}^{\prime}(s) A u(t-s) \mathrm{d} s=-k_{t}(0) A u(t)+\int_{0}^{\infty} k_{t}(s) A \partial_{t} u(t-s) \mathrm{d} s,
$$

equation (1.4) can be equivalently written as

$$
\partial_{t t} u(t)+A u(t)+\int_{0}^{\infty} k_{t}(s) A \partial_{t} u(t-s) \mathrm{d} s+f(u(t))=g .
$$

The main novelty of the model lies in the fact that the memory kernel depends itself on time. This feature allows to describe viscoelastic materials whose structural properties evolve over time. For instance, materials that undergo an aging process, which can be reasonably depicted as a loss of the elastic response. Indeed, the most interesting situation is when in the limit $t \rightarrow \infty$ the viscous effects become instantaneous. Describing a viscoelastic solid through a rheological model as a Maxwell element (i.e. a Hookean spring and a Newtonian dashpot sequentially connected) in parallel with a lone spring, this translates into a progressive stiffening of the spring in the Maxwell element, becoming eventually completely rigid (see the discussion in [4], see also [16]). In mathematical terms, this means that

$$
k_{t} \rightarrow m \delta_{0}, \quad m>0,
$$

namely, the integrated kernel $k_{t}(s)$ converges in the distributional sense to (a multiple of) the Dirac mass $\delta_{0}$ at zero as $t \rightarrow \infty$. In such a case, equation (1.7) formally collapses in

\footnotetext{
${ }^{1}$ The idea of describing the past values (i.e. the history) of $u$ via the introduction of an auxiliary variable goes back to the pioneering papers of C.M. Dafermos [9, 10].

${ }^{2}$ Here and in what follows, the prime denotes the derivative with respect to the internal variable $s$.
} 
the longtime into the Kelvin-Voigt model of viscoelasticity

$$
\partial_{t t} u(t)+A u(t)+m A \partial_{t} u(t)+f(u(t))=g,
$$

where the memory term disappears. In the terminology of Dautray and Lions [11], this represents the transition from a viscoelastic solid with "long memory" to a viscoelastic solid with "short memory", otherwise called of rate-type (see [12]). Notably, this convergence (at least at a formal level) occurs within the dynamics, and not just by letting some parameters go artificially to zero.

The theoretical challenge in [4] was to figure out a correct mathematical setting of the problem, in order to obtain an existence and uniqueness result. Indeed, the presence of a time-dependent kernel introduces essential difficulties in the analysis. As an example, to make a comparison with the standard theory, in the original work of Dafermos [9] (as well as in a number of later papers) the auxiliary variable $\eta$ is ruled by the differential equation

$$
\partial_{t} \eta^{t}(s)=-\partial_{s} \eta^{t}(s)+\partial_{t} u(t) .
$$

Contrary to the case of a memory kernel independent of time, here the problem is that the natural space of $\eta^{t}$ depends itself on $t$, reason why it is not even clear how to define the time-derivative of the variable. Nonetheless, as shown in [4], it is possible to give a good definition of solution within the theory of processes on time-dependent spaces, recently devised by Di Plinio et al. [14], and further developed in [6, 7, 8, 15].

The aim of the present work is to continue the analysis started in [4], studying the asymptotic properties of the solutions from a global-geometrical point of view.

- First, we establish the dissipative character of the dynamical process generated by (1.1)-(1.3), acting on suitable time-dependent phase spaces $\mathcal{H}_{t}$. By means of a recursive argument, we are able to find a constant $r_{0}>0$, independent of $t$ and $\tau$, such that the evolution at time $t$ of $\mathcal{H}_{\tau}$-bounded sets of initial data assigned at time $\tau<t$ has $\mathcal{H}_{t}$-norm less than $r_{0}$ as $t-\tau \rightarrow \infty$. In the terminology of [8], this entails the (uniform) timedependent absorbing set. Incidentally, the result applies (and is new) also for the classical case of a constant-in-time memory kernel, where an explicit stabilization estimate for the energy was not available. Indeed, e.g. in $[5,19]$, the absorbing set was eventually recovered as a byproduct of the global attractor, which in turn was obtained relying on the existence of a Lyapunov functional. Instead, direct energy estimates have two advantages: from the one side, the actual entering time of the solutions into the absorber can be calculated, from the other side the known results on the viscoelastic model can be extended, allowing for instance the presence of a time-dependent forcing term, that destroys the gradient system structure of the equation.

- The second step towards a more refined analysis is showing that the dynamical process possesses the so-called time-dependent global attractor. Loosely speaking, this is the smallest family of $t$-labeled sets $A_{t}$ able to attract bounded sets of initial data in a pullback sense. Besides, we will prove the optimal regularity of such a family. The existence of the time-dependent global attractor, which for the model under consideration turns out to be also invariant under the action of the process, provides a complete characterization of the regime behavior of the solutions.

- The final goal is to understand what happens in the limit $k_{t} \rightarrow m \delta_{0}$. In that situation, the convergence of the original problem to (1.8) turns out to be not only formal. Indeed, 
we will show that the $t$-sections $A_{t}$ of the time-dependent global attractor satisfy in a suitable sense the relation

$$
A_{t} \rightarrow \hat{A}, \quad \text { as } t \rightarrow \infty
$$

where $\hat{A}$ is the global attractor of the semigroup generated by (1.8), as defined in the classical books $[1,22,23,32]$. This provides a rigorous proof of the closeness of (1.1)-(1.3) to the Kelvin-Voigt model when the integrated kernel $k_{t}$ approaches the Dirac mass at zero as $t \rightarrow \infty$.

Outline of the paper. Under the general assumptions of the next Section 2, we first recall the main result of [4] on the generation of the time-dependent process of solutions. This is done in Section 3. Our results on the dissipative character of the process are stated in Section 4. The subsequent Sections 5-9 are devoted to their proofs. Namely, in Section 5 we introduce some auxiliary energy functionals, establishing suitable integral inequalities (the proof of one of which being quite long and technical, and therefore postponed in the Appendix A). In Section 6 we prove the existence of the time-dependent absorbing set, while the proofs concerning the time-dependent global attractor are given in Section 7 (existence), and Section 8 (regularity). Here a key ingredient is a Gronwall-type lemma in integral form, discussed in the Appendix B. In Section 9 we show the asymptotic closeness to the Kelvin-Voigt model when the kernel approaches the Dirac mass. In the final Section 10 we dwell on two particular memory kernels of physical interest complying with our general assumptions.

Notation. For $\sigma \in \mathbb{R}$, we define the compactly nested Hilbert spaces

$$
\mathrm{H}^{\sigma}=\mathfrak{D}\left(A^{\sigma / 2}\right), \quad\langle u, v\rangle_{\sigma}=\left\langle A^{\sigma / 2} u, A^{\sigma / 2} v\right\rangle_{\mathrm{H}} .
$$

Throughout the paper, the index $\sigma$ will be always omitted whenever zero. The symbol $\langle\cdot, \cdot\rangle$ will also be used to denote the duality pairing between $\mathrm{H}^{\sigma}$ and its dual space $\mathrm{H}^{-\sigma}$. Then, for every fixed time $t$, we introduce the weighted $L^{2}$-spaces, hereafter called memory spaces,

$$
\mathcal{M}_{t}^{\sigma}=L_{\mu_{t}}^{2}\left(\mathbb{R}^{+} ; \mathrm{H}^{\sigma+1}\right), \quad\langle\eta, \xi\rangle_{\mathcal{M}_{t}^{\sigma}}=\int_{0}^{\infty} \mu_{t}(s)\langle\eta(s), \xi(s)\rangle_{\sigma+1} \mathrm{~d} s .
$$

We will also consider the linear operator acting on $\mathcal{M}_{t}^{\sigma}$

$$
\mathbb{T}_{t} \eta=-\eta^{\prime} \quad \text { with domain } \mathfrak{D}\left(\mathbb{T}_{t}\right)=\left\{\eta \in \mathcal{M}_{t}^{\sigma}: \eta^{\prime} \in \mathcal{M}_{t}^{\sigma}, \eta(0)=0\right\} .
$$

Finally, we define the extended memory spaces

$$
\mathcal{H}_{t}^{\sigma}=\mathrm{H}^{\sigma+1} \times \mathrm{H}^{\sigma} \times \mathcal{M}_{t}^{\sigma},
$$

endowed with the usual product norm. For any $r \geq 0$, we will denote by

$$
\mathbb{B}_{t}^{\sigma}(r)=\left\{z \in \mathcal{H}_{t}^{\sigma}:\|z\|_{\mathcal{H}_{t}^{\sigma}} \leq r\right\}
$$

the closed $r$-ball about zero of $\mathcal{H}_{t}^{\sigma}$.

\section{General Assumptions}

We begin to stipulate our assumptions on the external force $g$, the nonlinear term $f(u)$ and the memory kernel $\mu_{t}$. 
2.1. Assumptions on $\boldsymbol{g}$ and $\boldsymbol{f}$. Let $g \in \mathrm{H}$ be independent of time, and let $f \in \mathcal{C}^{2}(\mathbb{R})$, with $f(0)=0$, satisfy for some $c \geq 0$

$$
\left|f^{\prime \prime}(u)\right| \leq c(1+|u|)
$$

along with the dissipation condition

$$
\liminf _{|u| \rightarrow \infty} f^{\prime}(u)>-\lambda_{1},
$$

$\lambda_{1}>0$ being the first eigenvalue of $A$. In particular, (2.2) easily implies the relations

$$
\begin{aligned}
& 2\langle F(u), 1\rangle \geq-(1-\theta)\|u\|_{1}^{2}-c_{f}, \\
& 2\langle f(u), u\rangle \geq 2\langle F(u), 1\rangle-(1-\theta)\|u\|_{1}^{2}-c_{f},
\end{aligned}
$$

for some $0<\theta \leq 1$ and $c_{f} \geq 0$, where

$$
F(u)=\int_{0}^{u} f(s) \mathrm{d} s .
$$

Remark 2.1. If $f$ is essentially monotone, i.e.

$$
\inf _{u \in \mathbb{R}} f^{\prime}(u)>-\lambda_{1}
$$

then $c_{f}=0$ in formulae $(2.3)-(2.4)$.

2.2. Assumptions on the memory kernel. The map

$$
(t, s) \mapsto \mu_{t}(s): \mathbb{R} \times \mathbb{R}^{+} \rightarrow \mathbb{R}^{+}
$$

satisfies the following axioms.

(M1) For every fixed $t \in \mathbb{R}$, the map $s \mapsto \mu_{t}(s)$ is nonincreasing, absolutely continuous and summable. We denote the total mass of $\mu_{t}$ by

$$
\kappa(t)=\int_{0}^{\infty} \mu_{t}(s) \mathrm{d} s .
$$

(M2) For every $\tau \in \mathbb{R}$, there exists a function $K_{\tau}:[\tau, \infty) \rightarrow \mathbb{R}^{+}$, summable on any interval $[\tau, T]$, such that

$$
\mu_{t}(s) \leq K_{\tau}(t) \mu_{\tau}(s)
$$

for every $t \geq \tau$ and every $s>0$.

(M3) For almost every fixed $s>0$, the map $t \mapsto \mu_{t}(s)$ is differentiable for all $t \in \mathbb{R}$, and $^{3}$

$$
(t, s) \mapsto \mu_{t}(s) \in L^{\infty}(\mathcal{K}) \quad \text { and } \quad(t, s) \mapsto \dot{\mu}_{t}(s) \in L^{\infty}(\mathcal{K})
$$

for every compact set $\mathcal{K} \subset \mathbb{R} \times \mathbb{R}^{+}$.

(M4) There exists $\delta>0$ such that

$$
\dot{\mu}_{t}(s)+\mu_{t}^{\prime}(s)+\delta \kappa(t) \mu_{t}(s) \leq 0
$$

for every $t \in \mathbb{R}$ and almost every $s>0$.

(M5) The function $t \mapsto \kappa(t)$ fulfills

$$
\inf _{t \in \mathbb{R}} \kappa(t)>0
$$

\footnotetext{
${ }^{3}$ Here and in what follows, the dot denotes the derivative with respect to time.
} 
(M6) The function $t \mapsto \dot{\mu}_{t}(s)$ satisfies the uniform integral estimate

$$
\sup _{t \in \mathbb{R}} \frac{1}{[\kappa(t)]^{2}} \int_{0}^{\infty}\left|\dot{\mu}_{t}(s)\right| \mathrm{d} s<\infty .
$$

(M7) For every $t \in \mathbb{R}$, the function $s \mapsto \mu_{t}(s)$ is bounded about zero, with

$$
\sup _{t \in \mathbb{R}} \frac{\mu_{t}(0)}{[\kappa(t)]^{2}}<\infty \text {. }
$$

(M8) For every $a<b \in \mathbb{R}$, there exists $\nu>0$ such that

$$
\int_{\nu}^{1 / \nu} \mu_{t}(s) \mathrm{d} s \geq \frac{\kappa(t)}{2}
$$

for every $t \in[a, b]$.

2.3. About axioms (M1)-(M8). Axioms (M1)-(M4) have been introduced in [4], and turn out to be sufficient in order to obtain a well-posedness result (see the next Theorem 3.2). Actually, such a result in [4] holds by replacing (M4) with the weaker $(\mathbf{M} 4)^{\prime}$ There exists a function $M: \mathbb{R} \rightarrow \mathbb{R}^{+}$, bounded on bounded intervals, such that

$$
\dot{\mu}_{t}(s)+\mu_{t}^{\prime}(s) \leq M(t) \mu_{t}(s)
$$

for every $t \in \mathbb{R}$ and almost every $s>0$.

Note that in the classical case where the kernel does not depend on $t$ (i.e. $\mu_{t}=\mu$ for all $t$ ), axiom (M4) boils down to the well-known assumption

$$
\mu^{\prime}(s)+\delta \mu(s) \leq 0,
$$

devised in [9] and commonly adopted in the literature thereafter. Instead, the role of (M5)-(M8) is more technical, and it will become clear in the Appendix B. Actually, it is also possible to treat the case of kernels which are unbounded about zero, by weakening axiom (M7). We prefer to avoid such a choice here, which would introduce annoying (and unnecessary) complications, see e.g. [25]. We also point out that we are considering kernels that do not vanish on $\mathbb{R}^{+}$, modeling the so-called infinite delay case. However, without changes in the proofs, our analysis apply as well to the finite delay case, namely, when $\mu_{t}(s)$ becomes identically zero for $s$ large enough.

We conclude with some immediate consequences of the axioms that will be useful in the course of the investigation.

- In the light of (M1), for every fixed $t$ the function $s \mapsto \mu_{t}(s)$ is differentiable almost everywhere with $\mu_{t}^{\prime} \leq 0$. In particular (see e.g. [21]),

$$
\left\langle\mathbb{T}_{t} \eta, \eta\right\rangle_{\mathcal{M}_{t}^{\sigma}}=\frac{1}{2} \int_{0}^{\infty} \mu_{t}^{\prime}(s)\|\eta(s)\|_{\sigma+1}^{2} \mathrm{~d} s \leq 0, \quad \forall \eta \in \mathfrak{D}\left(\mathbb{T}_{t}\right) .
$$

This tells that $\mathbb{T}_{t}$ is a dissipative operator. Indeed, $\mathbb{T}_{t}$ turns out to be the infinitesimal generator of the right-translation semigroup on $\mathcal{M}_{t}^{\sigma}$.

- For every $\sigma \in \mathbb{R}$ and every $t>\tau$, axiom (M2) entails

$$
\|\eta\|_{\mathcal{M}_{t}^{\sigma}}^{2} \leq K_{\tau}(t)\|\eta\|_{\mathcal{M}_{\tau}^{\sigma}}^{2}, \quad \forall \eta \in \mathcal{M}_{\tau}^{\sigma}
$$

providing the continuous embedding $\mathcal{M}_{\tau}^{\sigma} \subset \mathcal{M}_{t}^{\sigma}$, hence $\mathcal{H}_{\tau}^{\sigma} \subset \mathcal{H}_{t}^{\sigma}$. In particular, $\mathbb{T}_{t} \supset \mathbb{T}_{\tau}$, i.e. the operators $\left\{\mathbb{T}_{t}\right\}_{t \geq \tau}$ are increasingly nested extensions of each other. 


\section{The Process on Time-Dependent Spaces}

Let us begin with the definition of weak solution from [4].

Definition 3.1. Let $T>\tau \in \mathbb{R}$, and let $z_{\tau}=\left(u_{\tau}, v_{\tau}, \eta_{\tau}\right) \in \mathcal{H}_{\tau}$ be a fixed vector. A function

$$
z(t)=\left(u(t), \partial_{t} u(t), \eta^{t}\right) \in \mathcal{H}_{t} \quad \text { for a.e. } t \in[\tau, T]
$$

is a solution to problem (1.1)-(1.3) on the time-interval $[\tau, T]$ with initial datum $z_{\tau}$ if:

(i) $u \in L^{\infty}\left(\tau, T ; \mathrm{H}^{1}\right), \partial_{t} u \in L^{\infty}(\tau, T ; \mathrm{H}), \partial_{t t} u \in L^{1}\left(\tau, T ; \mathrm{H}^{-1}\right)$.

(ii) $u(\tau)=u_{\tau}, \partial_{t} u(\tau)=v_{\tau}$.

(iii) The function $\eta^{t}$ fulfills the representation formula (1.2).

(iv) The function $u(t)$ fulfills (1.1) in the weak sense, i.e.

$$
\left\langle\partial_{t t} u(t), \phi\right\rangle+\langle u(t), \phi\rangle_{1}+\int_{0}^{\infty} \mu_{t}(s)\left\langle\eta^{t}(s), \phi\right\rangle_{1} \mathrm{~d} s+\langle f(u(t)), \phi\rangle=\langle g, \phi\rangle
$$

for almost every $t \in[\tau, T]$ and every test $\phi \in \mathrm{H}^{1}$.

The main result of [4] is the generation of a process of solutions for problem (1.1)-(1.3). Recall that a two-parameter family of operators

$$
U(t, \tau): \mathcal{H}_{\tau} \rightarrow \mathcal{H}_{t}, \quad t \geq \tau,
$$

is called a processes on time-dependent spaces (see $[6,7,8,14,15])$ if

$\diamond U(\tau, \tau)$ is the identity map on $\mathcal{H}_{\tau}$ for every $\tau$;

$\diamond U(t, \tau) U(\tau, s)=U(t, s)$ for every $t \geq \tau \geq s$.

This follows from (see [4])

Theorem 3.2. For every $T>\tau \in \mathbb{R}$ and every initial datum $z_{\tau}=\left(u_{\tau}, v_{\tau}, \eta_{\tau}\right) \in \mathcal{H}_{\tau}$, problem (1.1)-(1.3) admits a unique solution

$$
z(t)=\left(u(t), \partial_{t} u(t), \eta^{t}\right)=U(t, \tau) z_{\tau}
$$

on the interval $[\tau, T]$. Besides, $u \in \mathcal{C}\left([\tau, T], \mathrm{H}^{1}\right) \cap \mathcal{C}^{1}([\tau, T], \mathrm{H}), \eta^{t} \in \mathcal{M}_{t}$ for every $t$, and

$$
\sup _{t \in[\tau, T]}\left\|U(t, \tau) z_{\tau}\right\|_{\mathcal{H}_{t}}<C,
$$

for some $C>0$ depending only on $T, \tau$ and the $\mathcal{H}_{\tau}$-norm of $z_{\tau}$. Moreover, the map

$$
z_{\tau} \mapsto U(t, \tau) z_{\tau}
$$

is (locally) Lipschitz from $\mathcal{H}_{\tau}$ to $\mathcal{H}_{t}$, uniformly with respect to $t \in[\tau, T]$.

For a given $z_{\tau} \in \mathcal{H}_{\tau}$, we agree to define the energy of the solution $U(t, \tau) z_{\tau}$ as

$$
\mathcal{E}(t, \tau)=\frac{1}{2}\left\|U(t, \tau) z_{\tau}\right\|_{\mathcal{H}_{t}}^{2} .
$$




\section{Statements of the Results}

4.1. Dissipativity. First, we discuss the dissipative character of the process. Mathematically speaking, this means that $U(t, \tau)$ possesses a time-dependent absorbing set, as defined in [8] (see also $[14,15])$.

Definition 4.1. A family $\mathfrak{B}=\left\{B_{t}\right\}_{t \in \mathbb{R}}$ is called a (uniform) time-dependent absorbing set if it is uniformly bounded, i.e.

$$
\sup _{t \in \mathbb{R}}\left\|B_{t}\right\|_{\mathcal{H}_{t}}<\infty
$$

and, for every $R>0$, there exists an elapsed entering time $\tau_{\mathrm{e}}=\tau_{\mathrm{e}}(R) \geq 0$ such that

$$
t-\tau \geq \tau_{\mathrm{e}} \Rightarrow U(t, \tau) \mathbb{B}_{\tau}(R) \subset B_{t} .
$$

The existence of the time-dependent absorbing set is an immediate consequence of the following result.

Theorem 4.2. There exist constants $\omega>0, R_{0} \geq 0$ and an increasing positive function $\mathcal{Q}$, all independent of $t \geq \tau$, such that

$$
\mathcal{E}(t, \tau) \leq \mathcal{Q}(R) \mathrm{e}^{-\omega(t-\tau)}+R_{0}
$$

whenever $\mathcal{E}(\tau, \tau) \leq R$.

Indeed, after Theorem 4.2, Definition 4.1 applies by merely taking

$$
B_{t}=\mathbb{B}_{t}\left(r_{0}\right) \quad \text { with } \quad r_{0}>\sqrt{2 R_{0}} .
$$

In absence of a forcing term, and for $f$ essentially monotone, the theorem holds with $R_{0}=0$, yielding the exponential decay of the energy.

Corollary 4.3. If in addition $g=0$ and $f$ satisfies (2.5), then

$$
\mathcal{E}(t, \tau) \leq \mathcal{Q}(R) \mathrm{e}^{-\omega(t-\tau)},
$$

whenever $\mathcal{E}(\tau, \tau) \leq R$.

4.2. The global attractor. We then deepen the longterm analysis of the system, looking for the time-dependent attractor. This is the object characterizing the regime behavior of a process defined on a time-dependent family of spaces $[8,6,14,15]$.

Definition 4.4. The time-dependent global attractor for $U(t, \tau)$ is the smallest family $\mathfrak{A}=\left\{A_{t}\right\}_{t \in \mathbb{R}}$ with the following properties:

(i) Each section $A_{t}$ is compact in $\mathcal{H}_{t}$.

(ii) $\mathfrak{A}$ is pullback attracting, namely, it is uniformly bounded and the limit ${ }^{4}$

$$
\lim _{\tau \rightarrow-\infty}\left[\operatorname{dist}_{\mathcal{H}_{t}}\left(U(t, \tau) C_{\tau}, A_{t}\right)\right]=0
$$

holds for every uniformly bounded family $\mathfrak{C}=\left\{C_{t}\right\}_{t \in \mathbb{R}}$ and every $t \in \mathbb{R}$.

The existence of the (invariant) time-dependent global attractor for our problem reads as follows.

\footnotetext{
${ }^{4}$ We denote the Hausdorff semidistance of two (nonempty) sets $B, C \subset \mathcal{H}_{t}$ by$$
\operatorname{dist}_{\mathcal{H}_{t}}(B, C)=\sup _{x \in B} \inf _{y \in C}\|x-y\|_{\mathcal{H}_{t}} \text {. }
$$ 
Theorem 4.5. The process $U(t, \tau): \mathcal{H}_{\tau} \rightarrow \mathcal{H}_{t}$ possesses the time-dependent global attractor $\mathfrak{A}=\left\{A_{t}\right\}_{t \in \mathbb{R}}$. Besides, the attractor is invariant, i.e.

$$
U(t, \tau) A_{\tau}=A_{t}, \quad \forall t \geq \tau .
$$

According to [6, Theorem 3.2], the invariant time-dependent global attractor is characterized as the set of all complete bounded trajectories (CBT) of the process, that is,

$$
A_{t}=\{z(t): z \operatorname{CBT} \text { of } U(t, \tau)\}
$$

where a CBT of $U(t, \tau)$ is a map

$$
t \mapsto z(t)=\left(u(t), \partial_{t} u(t), \eta^{t}\right) \in \mathcal{H}_{t}
$$

satisfying

$$
\sup _{t \in \mathbb{R}}\|z(t)\|_{\mathcal{H}_{t}}<\infty \quad \text { and } \quad z(t)=U(t, \tau) z(\tau) \quad \forall t \geq \tau \in \mathbb{R} .
$$

Corollary 4.6. Given any $\mathrm{CBT}\left(u, \partial_{t} u, \eta\right)$, the equality

$$
\eta^{t}(s)=u(t-s)-u(t)
$$

holds for every $t \in \mathbb{R}$ and every $s>0$. In particular, (1.1)-(1.2) take the form (1.4).

Indeed, being $\left(u, \partial_{t} u, \eta\right)$ a $\mathrm{CBT}$, for any fixed $t>\tau$ we readily see from (1.2) that such an equality is true for all $0<s \leq t-\tau$, and letting $\tau \rightarrow-\infty$ the claim follows.

4.3. Regularity. The next theorem is concerned with the regularity of the attractor.

Theorem 4.7. The sections of the time-dependent global attractor $\mathfrak{A}=\left\{A_{t}\right\}_{t \in \mathbb{R}}$ (belong to and) are uniformly bounded in $\mathcal{H}_{t}^{1}$, namely,

$$
\sup _{t \in \mathbb{R}}\left\|A_{t}\right\|_{\mathcal{H}_{t}^{1}}<\infty .
$$

Remark 4.8. For any $\mathrm{CBT}\left(u, \partial_{t} u, \eta\right)$, equality (4.1) together with the regularity of Theorem 4.7 ensure that

$$
\eta^{t} \in \mathfrak{D}\left(\mathbb{T}_{t}\right), \quad \forall t \in \mathbb{R} .
$$

Besides, the formal integration by parts (1.6), and so the passage from (1.4) to (1.7), becomes rigorous. Thus, the function $u(t)$ satisfies (1.7) in the weak sense for almost every $t \in \mathbb{R}$.

4.4. Recovering Kelvin-Voigt. We finally discuss the case when $k_{t} \rightarrow m \delta_{0}$ for some $m>0$, that is, ${ }^{5}$

$$
\lim _{t \rightarrow \infty} \int_{\varepsilon}^{\infty} k_{t}(s) \mathrm{d} s= \begin{cases}m & \text { if } \varepsilon=0 \\ 0 & \text { if } \varepsilon>0\end{cases}
$$

Accordingly, in the longtime our problem collapses into the Kelvin-Voigt model of viscoelastic solids (1.8).

It is well-known that equation (1.8), often referred to as strongly damped wave equation, generates a $\mathcal{C}_{0}$-semigroup of solutions

$$
S(t): \mathrm{H}^{1} \times \mathrm{H} \rightarrow \mathrm{H}^{1} \times \mathrm{H}
$$

\footnotetext{
${ }^{5}$ Condition $(4.2)$ is the same as saying that the measure $k_{t}(s) \mathrm{d} s$ on $[0, \infty)$ converges weakly to the measure $m \delta_{0}$.
} 
possessing the global attractor $\hat{A}$ in the classical sense. Besides, $\hat{A}$ is a bounded subset of $\mathrm{H}^{2} \times \mathrm{H}^{1}$, and coincides with the sections at (any) time $t_{0} \in \mathbb{R}$ of the set of all CBT of $S(t)$ (see, e.g. [2, 17, 20, 24, 27, 28, 33]). Namely, for any fixed $t_{0} \in \mathbb{R}$,

$$
\hat{A}=\left\{\hat{z}\left(t_{0}\right): \hat{z} \text { CBT of } S(t)\right\} \text {. }
$$

Recall that a CBT of the semigroup $S(t)$ is a map (see [23])

$$
t \mapsto \hat{z}(t)=\left(\hat{u}(t), \partial_{t} \hat{u}(t)\right) \in \mathrm{H}^{1} \times \mathrm{H}
$$

satisfying

$$
\sup _{t \in \mathbb{R}}\|\hat{z}(t)\|_{\mathrm{H}^{1} \times \mathrm{H}}<\infty \quad \text { and } \quad \hat{z}(t+\tau)=S(t) \hat{z}(\tau) \quad \forall t \geq 0, \forall \tau \in \mathbb{R} .
$$

Our last theorem establishes the closeness of the longterm dynamics of (1.1)-(1.3) to the one of the "limit problem" (1.8) when $k_{t} \rightarrow m \delta_{0}$.

Theorem 4.9. Let (4.2) hold. Then, for any sequence $\left(u_{n}, \partial_{t} u_{n}, \eta_{n}\right)$ of CBT of $U(t, \tau)$ and any $t_{n} \rightarrow \infty$, there exists a $\mathrm{CBT}\left(\hat{u}, \partial_{t} \hat{u}\right)$ of $S(t)$ such that the convergence

$$
\sup _{t \in[-T, T]}\left[\left\|u_{n}\left(t+t_{n}\right)-\hat{u}(t)\right\|_{\mathrm{H}^{1}}+\left\|\partial_{t} u_{n}\left(t+t_{n}\right)-\partial_{t} \hat{u}(t)\right\|_{\mathrm{H}}\right] \rightarrow 0
$$

holds up to a subsequence as $n \rightarrow \infty$ for every $T>0$.

Defining the canonical projection from $\mathcal{H}_{t}$ onto $\mathrm{H}^{1} \times \mathrm{H}$ by $\mathbb{P}_{t}(u, v, \eta)=(u, v)$, the theorem above produces an immediate corollary.

Corollary 4.10. If (4.2) holds, then we have the convergence

$$
\lim _{t \rightarrow \infty}\left[\operatorname{dist}_{\mathrm{H}^{2} \times \mathrm{H}^{1}}\left(\mathbb{P}_{t} A_{t}, \hat{A}\right)\right]=0 .
$$

Indeed, (4.3) says in particular that for every $t_{n} \rightarrow \infty$ the convergence

$$
\operatorname{dist}_{\mathrm{H}^{2} \times \mathrm{H}^{1}}\left(\mathbb{P}_{t_{n}} A_{t_{n}}, \hat{A}\right) \rightarrow 0
$$

holds (up to a subsequence) as $n \rightarrow \infty$. This is clearly enough to draw the desired conclusion.

The proofs of the results stated above will be carried out in the next Sections 5-9.

A word of warning. In the forthcoming proofs, we will denote by $C$ and $\mathcal{Q}$ a generic positive constant and a generic increasing positive function, respectively, both independent of $t \geq \tau$. We will use several times (possibly without explicit mention) the Young, Hölder and Poincaré inequalities, as well as the standard Sobolev embeddings, such as $\mathrm{H}^{1} \subset L^{6}(\Omega)$. Besides, we will perform several energy estimates, which are rigorously justified within the Galerkin approximation scheme detailed in [4].

\section{Some Auxiliary Functionals}

As we said in the Introduction, one of the main technical difficulties inherent in the formulation of our problem is that we do not (cannot) have a differential equation ruling the evolution of the additional variable $\eta^{t}$. As a direct consequence, we are unable to draw directly differential inequalities, essential to produce any kind of energy estimates. The strategy to overcome this obstacle is to produce suitable integral inequalities, that would hold if we had suitable differential estimates (which we don't). To this end, let $\tau \in \mathbb{R}$ 
be fixed and let $\left(p_{\tau}, q_{\tau}, \psi_{\tau}\right) \in \mathcal{H}_{\tau}$ be a sufficiently regular initial datum. We consider for $t>\tau$ the equation

$$
\partial_{t t} p(t)+A p(t)+\int_{0}^{\infty} \mu_{t}(s) A \psi^{t}(s) \mathrm{d} s+\gamma(t)=0 .
$$

Here, $\gamma$ is a certain forcing term (possibly depending on $p$ ), while

$$
\psi^{t}(s)= \begin{cases}p(t)-p(t-s), & s \leq t-\tau \\ \psi_{\tau}(s-t+\tau)+p(t)-p_{\tau}, & s>t-\tau .\end{cases}
$$

The equation is supplemented with the initial conditions

$$
\left\{\begin{array}{l}
p(\tau)=p_{\tau}, \\
\partial_{t} p(\tau)=q_{\tau}, \\
\psi^{\tau}=\psi_{\tau} .
\end{array}\right.
$$

Assuming that (5.1)-(5.3) admits a sufficiently regular global solution

$$
\left(p(t), \partial_{t} p(t), \psi^{t}\right) \in \mathcal{H}_{t}
$$

on $[\tau, \infty)$, we establish some crucial integral inequalities involving the triplet $\left(p, \partial_{t} p, \psi\right)$. The first one, implied by (5.2)-(5.3) only, comes from [4, Section 5].

Lemma 5.1. For all $\sigma \in[0,1]$ and every $b>a \geq \tau$, we have

$$
\left\|\psi^{b}\right\|_{\mathcal{M}_{b}^{\sigma}}^{2}-\int_{a}^{b} \int_{0}^{\infty}\left[\dot{\mu}_{t}(s)+\mu_{t}^{\prime}(s)\right]\left\|\psi^{t}(s)\right\|_{\sigma+1}^{2} \mathrm{~d} s \mathrm{~d} t \leq\left\|\psi^{a}\right\|_{\mathcal{M}_{a}^{\sigma}}^{2}+2 \int_{a}^{b}\left\langle\partial_{t} p(t), \psi^{t}\right\rangle_{\mathcal{M}_{t}^{\sigma}} \mathrm{d} t .
$$

Remark 5.2. On account of (M4), we deduce in particular the integrability on $[a, b]$ of the map

$$
t \mapsto \kappa(t)\left\|\psi^{t}\right\|_{\mathcal{M}_{t}^{\sigma}}^{2}
$$

In the next two lemmas we state two further integral inequalities, in terms of the auxiliary functionals

$$
\begin{aligned}
& \Phi(t)=2\left\langle p(t), \partial_{t} p(t)\right\rangle, \\
& \Psi(t)=-\frac{2}{\kappa(t)} \int_{0}^{\infty} \mu_{t}(s)\left\langle\psi^{t}(s), \partial_{t} p(t)\right\rangle \mathrm{d} s,
\end{aligned}
$$

which are easily seen to satisfy the estimate

$$
|\Phi(t)|+|\Psi(t)| \leq C\left[\|p(t)\|_{1}^{2}+\left\|\partial_{t} p(t)\right\|^{2}+\left\|\psi^{t}\right\|_{\mathcal{M}_{t}}^{2}\right] .
$$

Indeed, the less obvious control of $|\Psi|$ follows from the Hölder-type inequality (which will actually occur several times in the forthcoming calculations)

$$
\int_{0}^{\infty} \mu_{t}(s)\left\|\psi^{t}(s)\right\|_{1} \mathrm{~d} s \leq \sqrt{\kappa(t)}\left\|\psi^{t}\right\|_{\mathcal{M}_{t}}
$$

together with an application of (M5). 
Lemma 5.3. For every $b>a \geq \tau$ and every $\varpi \in(0,1]$, the functional $\Phi$ satisfies

$$
\begin{aligned}
\Phi(b)+(2-\varpi) \int_{a}^{b}\|p(t)\|_{1}^{2} \mathrm{~d} t \leq & \Phi(a)+2 \int_{a}^{b}\left\|\partial_{t} p(t)\right\|^{2} \mathrm{~d} t \\
& +\frac{1}{\varpi} \int_{a}^{b} \kappa(t)\left\|\psi^{t}\right\|_{\mathcal{M}_{t}}^{2} \mathrm{~d} t-2 \int_{a}^{b}\langle\gamma(t), p(t)\rangle \mathrm{d} t .
\end{aligned}
$$

Lemma 5.4. For every $b>a \geq \tau$ and every $\varpi \in(0,1]$, the functional $\Psi$ satisfies

$$
\begin{aligned}
\Psi(b)+\int_{a}^{b}\left\|\partial_{t} p(t)\right\|^{2} \mathrm{~d} t \leq & \Psi(a)-M \int_{a}^{b} \int_{0}^{\infty}\left[\dot{\mu}_{t}(s)+\mu_{t}^{\prime}(s)\right]\left\|\psi^{t}(s)\right\|_{1}^{2} \mathrm{~d} s \mathrm{~d} t \\
& +\varpi \int_{a}^{b}\|p(t)\|_{1}^{2} \mathrm{~d} t+\frac{C}{\varpi} \int_{a}^{b} \kappa(t)\left\|\psi^{t}\right\|_{\mathcal{M}_{t}}^{2} \mathrm{~d} t \\
& +\int_{a}^{b} \frac{2}{\kappa(t)} \int_{0}^{\infty} \mu_{t}(s)\left\langle\psi^{t}(s), \gamma(t)\right\rangle \mathrm{d} s \mathrm{~d} t .
\end{aligned}
$$

The positive constants $M$ and $C$ depend only on the structural assumptions on the memory kernel.

The proof of Lemma 5.4 is very technical and requires several approximation steps; it is therefore postponed in the Appendix B in full detail. On the contrary, the rather standard proof of the first lemma is reported here below for the reader's convenience.

Proof of Lemma 5.3. Multiplying equation (5.1) by $2 p$ in $\mathrm{H}$ we draw

$$
\frac{\mathrm{d}}{\mathrm{d} t} \Phi(t)-2\left\|\partial_{t} p(t)\right\|^{2}+2\|p(t)\|_{1}^{2}+2\langle\gamma(t), p(t)\rangle=-2 \int_{0}^{\infty} \mu_{t}(s)\left\langle\psi^{t}(s), p(t)\right\rangle_{1} \mathrm{~d} s .
$$

Estimating the right-hand side by

$$
-2 \int_{0}^{\infty} \mu_{t}(s)\langle\psi(s), p(t)\rangle_{1} \mathrm{~d} s \leq \varpi\|p(t)\|_{1}^{2}+\frac{1}{\varpi} \kappa(t)\left\|\psi^{t}\right\|_{\mathcal{M}_{t}}^{2},
$$

and integrating on $[a, b]$ the claim follows.

Finally, we tailor the inequalities of Lemmas 5.3 and 5.4 to the special case where

$$
\gamma=f(p)-g
$$

with $f$ and $g$ as in our problem.

Lemma 5.5. For the particular $\gamma$ above, the previous inequalities enhance to

$$
\begin{gathered}
\Phi(b)+\left(1+\frac{\theta}{2}\right) \int_{a}^{b}\|p(t)\|_{1}^{2} \mathrm{~d} t+2 \int_{a}^{b}\langle F(p(t)), 1\rangle \mathrm{d} t-2 \int_{a}^{b}\langle g, p(t)\rangle \mathrm{d} t \\
\leq \Phi(a)+2 \int_{a}^{b}\left\|\partial_{t} p(t)\right\|^{2} \mathrm{~d} t+\frac{2}{\theta} \int_{a}^{b} \kappa(t)\left\|\psi^{t}\right\|_{\mathcal{M}_{t}}^{2} \mathrm{~d} t+c_{f}(b-a),
\end{gathered}
$$


with $\theta$ and $c_{f}$ given by (2.4), and

$$
\begin{aligned}
\Psi(b)+\int_{a}^{b}\left\|\partial_{t} p(t)\right\|^{2} \mathrm{~d} t \leq & \Psi(a)-M \int_{a}^{b} \int_{0}^{\infty}\left[\dot{\mu}_{t}(s)+\mu_{t}^{\prime}(s)\right]\left\|\psi^{t}(s)\right\|_{1}^{2} \mathrm{~d} s \mathrm{~d} t \\
& +2 \varpi \int_{a}^{b}\|p(t)\|_{1}^{2} \mathrm{~d} t+\varpi\|g\|^{2}(b-a) \\
& +\frac{1}{\varpi} \int_{a}^{b} \mathcal{Q}\left(\|p(t)\|_{1}\right) \kappa(t)\left\|\psi^{t}\right\|_{\mathcal{M}_{t}}^{2} \mathrm{~d} t .
\end{aligned}
$$

Proof. Inequality (5.7) follows from Lemma 5.3 by choosing $\varpi=\theta / 2$ and exploiting (2.4).

In order to prove (5.8), we first note that, by (2.1) together with $f(0)=0$,

$$
\|\gamma\| \leq\|f(p)\|+\|g\| \leq \mathcal{Q}\left(\|p\|_{1}\right)\|p\|_{1}+\|g\| .
$$

Hence, making use of (M5),

$$
\begin{aligned}
& \frac{2}{\kappa(t)} \int_{0}^{\infty} \mu_{t}(s)\left\langle\psi^{t}(s), \gamma(t)\right\rangle \mathrm{d} s \\
& \quad \leq C\|\gamma(t)\| \int_{0}^{\infty} \mu_{t}(s)\left\|\psi^{t}(s)\right\| \mathrm{d} s \\
& \quad \leq C\left[\mathcal{Q}\left(\|p(t)\|_{1}\right)\|p(t)\|_{1}+\|g\|\right] \sqrt{\kappa(t)}\left\|\psi^{t}\right\|_{\mathcal{M}_{t}} \\
& \quad \leq \varpi\|p(t)\|_{1}^{2}+\varpi\|g\|^{2}+\frac{1}{\varpi} \mathcal{Q}\left(\|p(t)\|_{1}\right) \kappa(t)\left\|\psi^{t}\right\|_{\mathcal{M}_{t}}^{2} .
\end{aligned}
$$

Substituting the result in Lemma 5.4 we are done.

\section{Dissipativity: Proof of Theorem 4.2}

Throughout the section, let $\tau \in \mathbb{R}$ be an arbitrarily fixed initial time, and let $z_{\tau} \in \mathcal{H}_{\tau}$ be an arbitrary initial datum for which

$$
\mathcal{E}(\tau, \tau) \leq R, \quad \text { for some given } R \geq 0 .
$$

We preliminarily show that the energy of the system remains bounded.

Proposition 6.1. For every $t \geq \tau$ we have the estimate

$$
\mathcal{E}(t, \tau) \leq \mathcal{Q}(R)
$$

Proof. For $t \geq \tau$, we define the (Lyapunov) functional

$$
\mathcal{L}(t)=L(t)+\left\|\eta^{t}\right\|_{\mathcal{M}_{t}}^{2},
$$

where

$$
L(t)=\|u(t)\|_{1}^{2}+\left\|\partial_{t} u(t)\right\|^{2}+2\langle F(u(t)), 1\rangle-2\langle g, u(t)\rangle .
$$

Exploiting the inequalities (the first one being a consequence of (2.1))

$$
\begin{aligned}
2\langle F(u), 1\rangle & \leq C\left(1+\|u\|_{1}^{4}\right), \\
2|\langle g, u\rangle| & \leq \frac{\theta}{2}\|u\|_{1}^{2}+C\|g\|^{2},
\end{aligned}
$$

we deduce from (2.3) that

$$
\theta \mathcal{E}(t, \tau)-Q_{0} \leq \mathcal{L}(t) \leq \mathcal{Q}(\mathcal{E}(t, \tau))
$$


for some $Q_{0} \geq 0$. In particular,

$$
Q_{0}=0 \quad \text { if } \quad g=0 \text { and } c_{f}=0 .
$$

Testing equation (1.1) with $\partial_{t} u$ and integrating on $[a, b]$, we get

$$
L(b)+2 \int_{a}^{b}\left\langle\partial_{t} u(t), \eta^{t}\right\rangle_{\mathcal{M}_{t}} \mathrm{~d} t=L(a),
$$

for every $b>a \geq \tau$. On the other hand, an application of Lemma 5.1 with $\sigma=0$ yields

$$
\left\|\eta^{b}\right\|_{\mathcal{M}_{b}}^{2}-\int_{a}^{b} \int_{0}^{\infty}\left[\dot{\mu}_{t}(s)+\mu_{t}^{\prime}(s)\right]\left\|\eta^{t}(s)\right\|_{1}^{2} \mathrm{~d} s \mathrm{~d} t \leq\left\|\eta^{a}\right\|_{\mathcal{M}_{a}}^{2}+2 \int_{a}^{b}\left\langle\partial_{t} u(t), \eta^{t}\right\rangle_{\mathcal{M}_{t}} \mathrm{~d} t .
$$

Adding the two inequalities, we are led to

$$
\mathcal{L}(b)-\int_{a}^{b} \int_{0}^{\infty}\left[\dot{\mu}_{t}(s)+\mu_{t}^{\prime}(s)\right]\left\|\eta^{t}(s)\right\|_{1}^{2} \mathrm{~d} s \mathrm{~d} t \leq \mathcal{L}(a) .
$$

In particular, since by (M4) the integral in the left-hand side is positive,

$$
\mathcal{L}(t) \leq \mathcal{L}(\tau)
$$

and the desired conclusion follows from (6.1).

For $\varepsilon \in(0,1]$ to be suitably fixed later, we introduce the further functional

$$
\Lambda(t)=\mathcal{L}(t)+2 \varepsilon[\Phi(t)+4 \Psi(t)]+Q_{0},
$$

with $Q_{0}$ as in (6.1) and $\Phi, \Psi$ defined in Section 5 by the choice $\left(p, \partial_{t} p, \psi\right)=\left(u, \partial_{t} u, \eta\right)$. Due to (5.6) and (6.1), and by the proposition above, the inequality

$$
\frac{\theta}{2} \mathcal{E}(t, \tau) \leq \Lambda(t) \leq \mathcal{Q}(\mathcal{E}(t, \tau)) \leq \mathcal{Q}(R)
$$

is easily seen to hold, provided that $\varepsilon$ is small enough.

Lemma 6.2. There exist a constant $Q \geq 0$ (independent of $R$ ) and $\varepsilon=\varepsilon(R)>0$ small enough such that for every $b>a \geq \tau$

$$
\Lambda(b)+2 \varepsilon \int_{r}^{t} \Lambda(y) \mathrm{d} y \leq \Lambda(a)+\varepsilon Q(b-a) .
$$

If in addition $g=0$ and $c_{f}=0$, then $Q=0$.

Proof. From Proposition 6.1,

$$
\mathcal{Q}\left(\|u(t)\|_{1}\right) \leq \mathcal{Q}(R) .
$$

Hence, recalling that $\theta \leq 1$ and using (M5), collecting (5.7) and (5.8) with $\varpi=\theta / 32$ we infer that

$$
\begin{aligned}
& \Phi(b)+4 \Psi(b)+\int_{a}^{b} \mathcal{L}(t) \mathrm{d} t+\frac{\theta}{2} \int_{a}^{b} \mathcal{E}(t, \tau) \mathrm{d} t \\
& \leq \Phi(a)+4 \Psi(a)-4 M \int_{a}^{b} \int_{0}^{\infty}\left[\dot{\mu}_{t}(s)+\mu_{t}^{\prime}(s)\right]\left\|\eta^{t}(s)\right\|_{1}^{2} \mathrm{~d} s \mathrm{~d} t \\
& \quad+\mathcal{Q}(R) \int_{a}^{b} \kappa(t)\left\|\eta^{t}\right\|_{\mathcal{M}_{t}}^{2} \mathrm{~d} t+Q_{1}(b-a),
\end{aligned}
$$


for some $Q_{1} \geq 0$, which equals zero when $g=0$ and $c_{f}=0$. Therefore, taking into account (6.2), we arrive at

$$
\Lambda(b)+2 \varepsilon \int_{a}^{b} \Lambda(t) \mathrm{d} t+\mathfrak{I}_{1}+\mathfrak{I}_{2} \leq \Lambda(a)+\varepsilon Q(b-a),
$$

where $Q=2\left(Q_{0}+Q_{1}\right)$ and

$$
\begin{aligned}
& \mathfrak{I}_{1}=\varepsilon \int_{a}^{b}(\theta \mathcal{E}(t, \tau)-4 \varepsilon[\Phi(t)+4 \Psi(t)]) \mathrm{d} t \\
& \mathfrak{I}_{2}=-(1-8 \varepsilon M) \int_{a}^{b} \int_{0}^{\infty}\left[\dot{\mu}_{t}(s)+\mu_{t}^{\prime}(s)\right]\left\|\eta^{t}(s)\right\|_{1}^{2} \mathrm{~d} s \mathrm{~d} t-\varepsilon \mathcal{Q}(R) \int_{a}^{b} \kappa(t)\left\|\eta^{t}\right\|_{\mathcal{M}_{t}}^{2} \mathrm{~d} t .
\end{aligned}
$$

Observe that $Q=0$ if $g=0$ and $c_{f}=0$. The proof is finished if we show that $\mathfrak{I}_{1}$ and $\mathfrak{I}_{2}$ are nonnegative. Concerning $\mathfrak{I}_{1}$, this easily follows from (5.6) up to taking $\varepsilon$ sufficiently small. Coming to $\mathfrak{I}_{2}$, we first apply (M4) to get

$$
\mathfrak{I}_{2} \geq[\delta(1-8 \varepsilon M)-\varepsilon \mathcal{Q}(R)] \int_{a}^{b} \kappa(t)\left\|\eta^{t}\right\|_{\mathcal{M}_{t}}^{2} \mathrm{~d} t,
$$

up to taking $\varepsilon$ small enough such that $1-8 \varepsilon M>0$. A further reduction of $\varepsilon$, so that

$$
\delta(1-8 \varepsilon M) \geq \varepsilon \mathcal{Q}(R),
$$

yields the claim. Note that the obtained value of $\varepsilon$ depends on $R$.

Proofs of Theorem 4.2 and Corollary 4.3. After Lemma 6.2, we are in a position to apply the Gronwall-type Lemma B.1 in the Appendix B with $q_{1}=0$ and $q_{2}=\varepsilon Q$, to get

$$
\Lambda(t) \leq \Lambda(\tau) \mathrm{e}^{-\varepsilon(t-\tau)}+\frac{\varepsilon Q \mathrm{e}^{\varepsilon}}{1-\mathrm{e}^{-\varepsilon}}
$$

Therefore, by (6.3),

where

$$
\mathcal{E}(t, \tau) \leq \frac{\theta}{2} \Lambda(t) \leq \mathcal{Q}(R) \mathrm{e}^{-\varepsilon(t-\tau)}+R_{0}
$$

$$
R_{0}=\frac{\theta Q}{2} \sup _{\varepsilon \in(0,1]} \frac{\varepsilon \mathrm{e}^{\varepsilon}}{1-\mathrm{e}^{-\varepsilon}} .
$$

The constant $R_{0}$, which is independent of $R$, equals zero when $g=0$ and $c_{f}=0$. The latter occurs whenever (2.5) holds (see Remark 2.1). In fact, such a result is already enough in order to obtain the time-dependent absorbing set. However, by means of a simple trick, it is possible to show that the decay rate $\varepsilon=\varepsilon(R)$ is actually independent of $R$. This is particularly relevant in the case $R_{0}=0$, for it gives the exponential decay of the energy. To this end, we observe that there is an elapsed time $\tau_{\star}=\tau_{\star}(R) \geq 0$ such that

$$
\mathcal{E}\left(t_{\star}, \tau\right) \leq R_{0}+1 \quad \text { where } \quad t_{\star}=\tau_{\star}+\tau .
$$

Hence, for $t \geq t_{\star}$,

$$
\mathcal{E}(t, \tau)=\frac{1}{2}\left\|U\left(t, t_{\star}\right) U\left(t_{\star}, \tau\right) z_{\tau}\right\|_{\mathcal{H}_{t}}^{2} \leq \mathcal{Q}\left(R_{0}+1\right) \mathrm{e}^{-\varepsilon\left(t-t_{\star}\right)}+R_{0},
$$

where $\varepsilon=\varepsilon\left(R_{0}+1\right)$. On the other hand, if $t \in\left[\tau, t_{\star}\right]$,

$$
\mathcal{E}(t, \tau) \leq \mathcal{Q}(R)
$$


Then, collecting the two inequalities, for every $t \geq \tau$ we obtain

$$
\mathcal{E}(t, \tau) \leq \mathcal{Q}(R) \mathrm{e}^{-\varepsilon\left(t-t_{\star}\right)}+R_{0}=\mathcal{Q}(R) \mathrm{e}^{\varepsilon \tau_{\star}} \mathrm{e}^{-\varepsilon(t-\tau)}+R_{0}=\mathcal{Q}(R) \mathrm{e}^{-\varepsilon(t-\tau)}+R_{0} .
$$

In summary, the desired estimate holds by setting

$$
\omega=\varepsilon\left(R_{0}+1\right)
$$

which is now independent of $R$.

We conclude the section by showing the existence of a dissipation integral, which will be needed later.

Lemma 6.3. For every $\varepsilon \in(0,1]$ and every $b>a \geq \tau$,

$$
\int_{a}^{b}\left\|\partial_{t} u(t)\right\|^{2} \mathrm{~d} t \leq \varepsilon(b-a)+\frac{\mathcal{Q}(R)}{\varepsilon^{2}} .
$$

Proof. For $\varpi \in(0,1]$, we consider the functional

$$
\Upsilon(t)=\mathcal{L}(t)+\varpi^{2} \Psi(t)
$$

Exploiting (5.6), (5.8), (6.2) and Proposition 6.1, we deduce the integral inequality

$$
\varpi^{2} \int_{a}^{b}\left\|\partial_{t} u(t)\right\|^{2} \mathrm{~d} t+\mathfrak{I} \leq \varpi^{3} \mathcal{Q}(R)(b-a)+\Upsilon(a)-\Upsilon(b) \leq \varpi^{3} \mathcal{Q}(R)(b-a)+\mathcal{Q}(R),
$$

having set

$$
\mathfrak{I}=-\left(1-\varpi^{2} M\right) \int_{a}^{b} \int_{0}^{\infty}\left[\dot{\mu}_{t}(s)+\mu_{t}^{\prime}(s)\right]\left\|\eta^{t}(s)\right\|_{1}^{2} \mathrm{~d} s \mathrm{~d} t-\varpi \mathcal{Q}(R) \int_{a}^{b} \kappa(t)\left\|\eta^{t}\right\|_{\mathcal{M}_{t}}^{2} \mathrm{~d} t .
$$

Owing to (M4), up to possibly reducing $\varpi$,

$$
\mathfrak{I} \geq\left[\delta\left(1-\varpi^{2} M\right)-\varpi \mathcal{Q}(R)\right] \int_{a}^{b} \kappa(t)\left\|\eta^{t}\right\|_{\mathcal{M}_{t}}^{2} \mathrm{~d} t \geq 0
$$

Therefore,

$$
\int_{a}^{b}\left\|\partial_{t} u(t)\right\|^{2} \mathrm{~d} t \leq \varpi \mathcal{Q}(R)(b-a)+\frac{\mathcal{Q}(R)}{\varpi^{2}}
$$

If $\varepsilon>0$ is small enough, up to taking $\varpi=\varepsilon / \mathcal{Q}(R)$ we obtain the desired inequality, which then clearly holds for every $\varepsilon \in(0,1]$.

\section{The Global Attractor: Proof of Theorem 4.5}

We show that the process $U(t, \tau): \mathcal{H}_{\tau} \rightarrow \mathcal{H}_{t}$ possesses the time-dependent global attractor $\mathfrak{A}$. The proof leans on an abstract result devised in [8], saying that $\mathfrak{A}$ exists if and only if there is a pullback attracting family ${ }^{6} \mathfrak{C}=\left\{C_{t}\right\}_{t \in \mathbb{R}}$ whose sections $C_{t}$ are compact in $\mathcal{H}_{t}$. Such an attractor $\mathfrak{A}$ is invariant as well. Indeed, we know from [8, Theorem 5.6] that, whenever it exists, the time-dependent global attractor of a continuous-in-space process is always invariant. And in our case, in the light of Theorem 3.2, our process $U(t, \tau)$ is even Lipschitz continuous.

Accordingly, the aim is to find a pullback attracting family with compact sections. To this end, let $\mathfrak{B}=\left\{B_{t}\right\}_{t \in \mathbb{R}}$ be a time-dependent absorbing set for the process $U(t, \tau)$. In what follows, $\tau \in \mathbb{R}$ is an arbitrarily fixed starting time, and $z_{\tau} \in B_{\tau}$ an arbitrarily fixed

\footnotetext{
${ }^{6}$ The notion of pullback attracting family is given in Definition 4.4.
} 
initial datum. Throughout this section, the generic constant $C>0$ may depend on $\mathfrak{B}$, but is independent of $\tau$ and of the particular choice of $z_{\tau}$. By Proposition 6.1,

$$
\left\|U(t, \tau) z_{\tau}\right\|_{\mathcal{H}_{t}} \leq C, \quad \forall t \geq \tau
$$

Following a pretty standard procedure (see e.g. [13]), we split the nonlinearity $f$ into the sum

$$
f(u)=f_{0}(u)+f_{1}(u),
$$

where $f_{1} \in \mathcal{C}^{2}(\mathbb{R})$ is globally Lipschitz with $f_{1}(0)=0$, while $f_{0} \in \mathcal{C}^{2}(\mathbb{R})$ vanishes inside $[-1,1]$ and fulfills the inequalities

$$
\begin{aligned}
& \left|f_{0}^{\prime \prime}(u)\right| \leq C|u|, \\
& f_{0}^{\prime}(u) \geq 0 .
\end{aligned}
$$

Then, we decompose the solution $U(t, \tau) z_{\tau}$ as

$$
U(t, \tau) z_{\tau}=U_{0}(t, \tau) z_{\tau}+U_{1}(t, \tau) z_{\tau},
$$

where

$$
U_{0}(t, \tau) z_{\tau}=\left(v(t), \partial_{t} v(t), \xi^{t}\right) \quad \text { and } \quad U_{1}(t, \tau) z_{\tau}=\left(w(t), \partial_{t} w(t), \zeta^{t}\right)
$$

solve the problems

$$
\left\{\begin{array}{l}
\partial_{t t} v(t)+A v(t)+\int_{0}^{\infty} \mu_{t}(s) A \xi^{t}(s) \mathrm{d} s+f_{0}(v(t))=0 \\
U_{0}(\tau, \tau) z_{\tau}=z_{\tau}
\end{array}\right.
$$

where

$$
\xi^{t}(s)= \begin{cases}v(t)-v(t-s), & s \leq t-\tau \\ \xi_{\tau}(s-t+\tau)+v(t)-v_{\tau}, & s>t-\tau\end{cases}
$$

and

$$
\left\{\begin{array}{l}
\partial_{t t} w(t)+A w(t)+\int_{0}^{\infty} \mu_{t}(s) A \zeta^{t}(s) \mathrm{d} s+f_{0}(u(t))-f_{0}(v(t))+f_{1}(u(t))=g \\
U_{1}(\tau, \tau) z_{\tau}=0
\end{array}\right.
$$

where

$$
\zeta^{t}(s)= \begin{cases}w(t)-w(t-s), & s \leq t-\tau \\ \zeta_{\tau}(s-t+\tau)+w(t)-w_{\tau}, & s>t-\tau .\end{cases}
$$

Note that $U_{0}(t, \tau)$ is itself a process. Besides, due to (7.3), Corollary 4.3 applies as well to (7.4), providing the exponential decay

$$
\left\|U_{0}(t, \tau) z_{\tau}\right\|_{\mathcal{H}_{t}}^{2} \leq C \mathrm{e}^{-\omega(t-\tau)} .
$$

Lemma 7.1. For every $t \geq \tau$, we have the estimate

$$
\mathcal{E}_{1}(t)=\frac{1}{2}\left\|U_{1}(t, \tau) z_{\tau}\right\|_{\mathcal{H}_{t}^{1 / 3}}^{2} \leq C .
$$


Proof. For $t \geq \tau$, we consider the functional

$$
\mathcal{L}_{1}(t)=L_{1}(t)+\left\|\zeta^{t}\right\|_{\mathcal{M}_{t}^{1 / 3}}^{2}
$$

where

$$
L_{1}(t)=\|w(t)\|_{4 / 3}^{2}+\left\|\partial_{t} w(t)\right\|_{1 / 3}^{2}+2\left\langle\gamma(t), A^{1 / 3} w(t)\right\rangle
$$

having defined

$$
\gamma(t)=f_{0}(u(t))-f_{0}(v(t))+f_{1}(u(t))-g .
$$

Collecting (2.1), (7.1), (7.2) and (7.6), we learn that

$$
\|\gamma(t)\| \leq C
$$

and we readily deduce the controls

$$
\frac{3}{2} \mathcal{E}_{1}(t)-C \leq \mathcal{L}_{1}(t) \leq \frac{5}{2} \mathcal{E}_{1}(t)+C .
$$

A multiplication in $\mathrm{H}^{1 / 3}$ of the first equation of (7.5) by $2 \partial_{t} w$ gives

$$
\begin{aligned}
& \frac{\mathrm{d}}{\mathrm{d} t} L_{1}+2\left\langle\zeta, \partial_{t} w\right\rangle_{\mathcal{M}_{t}^{1 / 3}} \\
& \quad=2\left\langle\left[f_{0}^{\prime}(u)-f_{0}^{\prime}(v)\right] \partial_{t} u, A^{1 / 3} w\right\rangle+2\left\langle f_{0}^{\prime}(v) \partial_{t} w, A^{1 / 3} w\right\rangle+2\left\langle f_{1}^{\prime}(u) \partial_{t} u, A^{1 / 3} w\right\rangle .
\end{aligned}
$$

The estimate of the right-hand side is completely standard, by means of the (uniform) bounds (7.1), (7.2), (7.6), $\left|f_{1}^{\prime}\right| \leq C$, along with the Sobolev embeddings

$$
\mathrm{H}^{\sigma} \subset L^{6 /(3-2 \sigma)}(\Omega), \quad \sigma \in\left(0, \frac{3}{2}\right) .
$$

Namely,

$$
\begin{aligned}
2\left\langle\left[f_{0}^{\prime}(u)-f_{0}^{\prime}(v)\right] \partial_{t} u, A^{1 / 3} w\right\rangle & \leq C\left(1+\|u\|_{L^{6}}+\|v\|_{L^{6}}\right)\left\|\partial_{t} u\right\|\|w\|_{L^{18}}\left\|A^{1 / 3} w\right\|_{L^{18 / 5}} \\
& \leq C\left\|\partial_{t} u\right\|\|w\|_{4 / 3}^{2}, \\
2\left\langle f_{0}^{\prime}(v) \partial_{t} w, A^{1 / 3} w\right\rangle & \leq C\|v\|_{L^{6}}^{2}\left\|\partial_{t} w\right\|_{L^{18 / 7}}\left\|A^{1 / 3} w\right\|_{L^{18 / 5}} \\
& \leq C\|v\|_{1}^{2}\left(\|w\|_{4 / 3}^{2}+\left\|\partial_{t} w\right\|_{1 / 3}^{2}\right) \\
2\left\langle f_{1}^{\prime}(v) \partial_{t} u, A^{1 / 3} w\right\rangle & \leq C\left\|\partial_{t} u\right\|+C\left\|\partial_{t} u\right\|\|w\|_{4 / 3}^{2} .
\end{aligned}
$$

In summary, defining

$$
q(t)=\left\|\partial_{t} u(t)\right\|+\|v(t)\|_{1}^{2},
$$

and recalling (7.8), the right-hand side above is bounded by $C q+C q \mathcal{L}_{1}$. Note that, by Lemma 6.3 and (7.6),

$$
\int_{a}^{b}\|q(t)\| \mathrm{d} t \leq \varepsilon(b-a)+\frac{C}{\varepsilon^{3}}
$$

for every $b>a \geq \tau$ and every $\varepsilon \in(0,1]$. Hence, an integration on $[a, b]$ yields

$$
L_{1}(b)+2 \int_{a}^{b}\left\langle\partial_{t} w(t), \zeta^{t}\right\rangle_{\mathcal{M}_{t}^{1 / 3}} \mathrm{~d} t \leq L_{1}(a)+C \int_{a}^{b} q(t) \mathcal{L}_{1}(t) \mathrm{d} t+C \int_{a}^{b} q(t) \mathrm{d} t .
$$

At the same time, from Lemma 5.1 for $\sigma=1 / 3$,

$$
\left\|\zeta^{b}\right\|_{\mathcal{M}_{b}^{1 / 3}}^{2}-\int_{a}^{b} \int_{0}^{\infty}\left[\dot{\mu}_{t}(s)+\mu_{t}^{\prime}(s)\right]\left\|\zeta^{t}(s)\right\|_{4 / 3}^{2} \mathrm{~d} s \mathrm{~d} t \leq\left\|\zeta^{a}\right\|_{\mathcal{M}_{a}^{1 / 3}}^{2}+2 \int_{a}^{b}\left\langle\partial_{t} w(t), \zeta^{t}\right\rangle_{\mathcal{M}_{t}^{1 / 3}} \mathrm{~d} t .
$$


Adding the two integral inequalities, we end up with

$$
\begin{array}{r}
\mathcal{L}_{1}(b)-\int_{a}^{b} \int_{0}^{\infty}\left[\dot{\mu}_{t}(s)+\mu_{t}^{\prime}(s)\right]\left\|\zeta^{t}(s)\right\|_{4 / 3}^{2} \mathrm{~d} s \mathrm{~d} t \\
\leq \mathcal{L}_{1}(a)+C \int_{a}^{b} q(t) \mathcal{L}_{1}(t) \mathrm{d} t+C \int_{a}^{b} q(t) \mathrm{d} t .
\end{array}
$$

For $\varepsilon \in(0,1]$ to be chosen later, we introduce the further functional

$$
\Lambda_{1}(t)=\mathcal{L}_{1}(t)+2 \varepsilon[\Phi(t)+4 \Psi(t)]
$$

where $\Phi$ and $\Psi$ are defined as in Section 5, using the triplet

$$
\left(p, \partial_{t} p, \psi\right)=\left(A^{1 / 6} w, A^{1 / 6} \partial_{t} w, A^{1 / 6} \zeta\right)
$$

In particular, (5.6) now reads

$$
|\Phi(t)|+|\Psi(t)| \leq C \mathcal{E}_{1}(t)
$$

Consequently, owing to (7.8), for $\varepsilon$ sufficiently small $\Lambda_{1}$ is controlled by

$$
\mathcal{E}_{1}(t)-C \leq \Lambda_{1}(t) \leq 3 \mathcal{E}_{1}(t)+C .
$$

Exploiting Lemmas 5.3 and 5.4 with $\varpi=1 / 10$, we infer that

$$
\begin{aligned}
& \Phi(b)+4 \Psi(b)+\frac{3}{2} \int_{a}^{b}\|w(t)\|_{4 / 3}^{2} \mathrm{~d} t+2 \int_{a}^{b}\left\|\partial_{t} w(t)\right\|_{1 / 3}^{2} \mathrm{~d} t \\
& \leq \Phi(a)+4 \Psi(a)-4 M \int_{a}^{b} \int_{0}^{\infty}\left[\dot{\mu}_{t}(s)+\mu_{t}^{\prime}(s)\right]\left\|\zeta^{t}(s)\right\|_{4 / 3}^{2} \mathrm{~d} s \mathrm{~d} t+C \int_{a}^{b} \kappa(t)\left\|\zeta^{t}\right\|_{\mathcal{M}_{t}^{1 / 3}}^{2} \mathrm{~d} t \\
& \quad-2 \int_{a}^{b}\left\langle\gamma(t), A^{1 / 3} w(t)\right\rangle \mathrm{d} t+\int_{a}^{b} \frac{2}{\kappa(t)} \int_{0}^{\infty} \mu_{t}(s)\left\langle\gamma(t), A^{1 / 3} \zeta^{t}(s)\right\rangle \mathrm{d} s \mathrm{~d} t .
\end{aligned}
$$

This inequality, making use of (M5), (7.7) and (7.12), enhances to

$$
\begin{aligned}
& \Phi(b)+4 \Psi(b)+\int_{a}^{b} \Lambda_{1}(t) \mathrm{d} t \\
& \leq \Phi(a)+4 \Psi(a)-4 M \int_{a}^{b} \int_{0}^{\infty}\left[\dot{\mu}_{t}(s)+\mu_{t}^{\prime}(s)\right]\left\|\zeta^{t}(s)\right\|_{4 / 3}^{2} \mathrm{~d} s \mathrm{~d} t \\
& \quad+C \int_{a}^{b} \kappa(t)\left\|\zeta^{t}\right\|_{\mathcal{M}_{t}^{1 / 3}}^{2} \mathrm{~d} t+C(b-a),
\end{aligned}
$$

and taking into account (7.10)-(7.12) we arrive at

$$
\Lambda_{1}(b)+2 \varepsilon \int_{a}^{b} \Lambda_{1}(t) \mathrm{d} t+\mathfrak{I} \leq \Lambda_{1}(a)+C \int_{a}^{b} q(t) \Lambda_{1}(t) \mathrm{d} t+C \int_{a}^{b}[q(t)+1] \mathrm{d} t
$$

where

$$
\mathfrak{I}=-(1-8 \varepsilon M) \int_{a}^{b} \int_{0}^{\infty}\left[\dot{\mu}_{t}(s)+\mu_{t}^{\prime}(s)\right]\left\|\zeta^{t}(s)\right\|_{4 / 3}^{2} \mathrm{~d} s \mathrm{~d} t-\varepsilon C \int_{a}^{b} \kappa(t)\left\|\zeta^{t}\right\|_{\mathcal{M}_{t}^{1 / 3}}^{2} \mathrm{~d} t .
$$

Owing to (M4), we get

$$
\mathfrak{I} \geq[\delta(1-8 \varepsilon M)-\varepsilon C] \int_{a}^{b} \kappa(t)\left\|\zeta^{t}\right\|_{\mathcal{M}_{t}^{1 / 3}}^{2} \mathrm{~d} t
$$


which is nonnegative up to fixing $\varepsilon$ sufficiently small that

$$
\delta(1-8 \varepsilon M) \geq \varepsilon C .
$$

In conclusion,

$$
\Lambda_{1}(b)-\Lambda_{1}(a)+2 \varepsilon \int_{a}^{b} \Lambda_{1}(t) \mathrm{d} t \leq \int_{a}^{b} q_{1}(t) \Lambda_{1}(t) \mathrm{d} t+\int_{a}^{b} q_{2}(t) \mathrm{d} t
$$

with

$$
q_{1}(t)=C q(t) \quad \text { and } \quad q_{2}(t)=C q(t)+C .
$$

Keeping in mind (7.9), we can apply the Gronwall-type Lemma B.1. Since $\Lambda_{1}(\tau)=0$, this gives

$$
\Lambda_{1}(t) \leq C, \quad \forall t \geq \tau
$$

The claim then follows from (7.12).

Proof of Theorem 4.5. Since $\mathfrak{B}=\left\{B_{t}\right\}_{t \in \mathbb{R}}$ is a time-dependent absorbing set, collecting (7.6) and Lemma 7.1 we infer that the family of $\mathcal{H}_{t}^{1 / 3}$-balls

$$
\mathfrak{B}^{\star}=\left\{\mathbb{B}_{t}^{1 / 3}(r)\right\}_{t \in \mathbb{R}}
$$

is pullback attracting provided that $r>0$ is sufficiently large, for

$$
\operatorname{dist}_{\mathcal{H}_{t}}\left(U(t, \tau) B_{\tau}, \mathbb{B}_{t}^{1 / 3}(r)\right) \leq \sup _{z_{\tau} \in B_{\tau}}\left\|U_{0}(t, \tau) z_{\tau}\right\|_{\mathcal{H}_{t}} \leq C \mathrm{e}^{-\frac{\omega}{2}(t-\tau)}
$$

Unfortunately, this is not enough to conclude. Indeed, although closed balls of $\mathcal{H}_{t}^{1 / 3}$ are uniformly bounded ${ }^{7}$, they fail to be compact in $\mathcal{H}_{t}$, due to the lack of compactness of the embedding $\mathcal{M}_{t}^{1 / 3} \subset \mathcal{M}_{t}$ (see [29]). On the other hand, up to possibly enlarge $r$, it is possible to find, for every fixed $t$, a compact set $C_{t} \subset \mathbb{B}_{t}^{1 / 3}(r)$ such that $\mathfrak{C}=\left\{C_{t}\right\}_{t \in \mathbb{R}}$ is still pullback attracting. The argument goes word by word as in the proof of Lemma 7.2 in [5], where the same model is considered for a constant-in-time memory kernel, and is therefore omitted. Accordingly, the existence of the time-dependent global attractor $\mathfrak{A}=\left\{A_{t}\right\}_{t \in \mathbb{R}}$ is attained.

\section{Regularity: Proof of Theorem 4.7}

In the previous proof, we found a pullback attracting family $\mathfrak{C}=\left\{C_{t}\right\}_{t \in \mathbb{R}}$ whose sections $C_{t}$ are compact and uniformly bounded in $\mathcal{H}_{t}^{1 / 3}$. Since, due to the minimality property of the attractor, the inclusion $A_{t} \subset C_{t}$ holds for every $t \in \mathbb{R}$, it follows that

$$
\sup _{t \in \mathbb{R}}\left\|A_{t}\right\|_{\mathcal{H}_{t}^{1 / 3}}<\infty
$$

meaning that the sections $A_{t}$ are uniformly bounded in $\mathcal{H}_{t}^{1 / 3}$. The next step is to improve such a regularity. To this end, we exploit once more the decomposition strategy discussed above, but for initial data on the attractor. Namely, for arbitrarily fixed $\tau \in \mathbb{R}$ and $z_{\tau} \in A_{\tau}$, let us write

$$
U(t, \tau) z_{\tau}=U_{0}(t, \tau) z_{\tau}+U_{1}(t, \tau) z_{\tau}
$$

where $U_{0}(t, \tau)$ and $U_{1}(t, \tau)$ satisfy (7.4) and (7.5), respectively, with

$$
f_{0}(u)=0 \quad \text { and } \quad f_{1}(u)=f(u) .
$$

\footnotetext{
${ }^{7}$ Note that, for any $\sigma>0$, the embedding constant of $\mathcal{M}_{t}^{\sigma} \subset \mathcal{M}_{t}$ is independent of $t$.
} 
In particular, Corollary 4.3 applies to $U_{0}(t, \tau)$, yielding the exponential decay

$$
\left\|U_{0}(t, \tau) z_{\tau}\right\|_{\mathcal{H}_{t}}^{2} \leq C \mathrm{e}^{-\omega(t-\tau)} .
$$

Here and in what follows, the generic constant $C>0$ depends only on the attractor $\mathfrak{A}$ (and is independent of $\tau$ and $z_{\tau} \in A_{\tau}$ ).

Lemma 8.1. For every $t \geq \tau$, we have the estimate

$$
\mathcal{E}_{2}(t)=\frac{1}{2}\left\|U_{1}(t, \tau) z_{\tau}\right\|_{\mathcal{H}_{t}^{1}}^{2} \leq C
$$

Proof. We just sketch the proof, which is completely analogous to the one of Lemma 7.1. By standard multiplications and Lemma 5.1 with $\sigma=1$, the functional

$$
\mathcal{L}_{2}(t)=\|w(t)\|_{2}^{2}+\left\|\partial_{t} w(t)\right\|_{1}^{2}+\left\|\zeta^{t}\right\|_{\mathcal{M}_{t}^{1}}^{2}+2\langle f(u(t)), A w(t)\rangle
$$

is shown to fulfill the integral inequality

$$
\begin{gathered}
\mathcal{L}_{2}(b)-\int_{a}^{b} \int_{0}^{\infty}\left[\dot{\mu}_{t}(s)+\mu_{t}^{\prime}(s)\right]\left\|\zeta^{t}(s)\right\|_{2}^{2} \mathrm{~d} s \mathrm{~d} t \\
\leq \mathcal{L}_{2}(a)+\frac{\varepsilon}{2} \int_{a}^{b}\|w(t)\|_{2}^{2} \mathrm{~d} t+\frac{C}{\varepsilon}(b-a)
\end{gathered}
$$

for every $b>a \geq \tau$ and every $\varepsilon \in(0,1]$. Here, the only difference with respect to the previous proof is the control

$$
2\left\langle f^{\prime}(u) \partial_{t} u, A w\right\rangle \leq C\left(1+\|u\|_{L^{18}}\right)\left\|\partial_{t} u\right\|_{L^{18 / 7}}\|A w\| \leq \frac{\varepsilon}{2}\|w\|_{2}^{2}+\frac{C}{\varepsilon} .
$$

Then we introduce the functional

$$
\Lambda_{2}(t)=\mathcal{L}_{2}(t)+2 \varepsilon[\Phi(t)+4 \Psi(t)]
$$

where $\Phi$ and $\Psi$ are defined as in Section 5, using the triplet

$$
\left(p, \partial_{t} p, \psi\right)=\left(A^{1 / 2} w, A^{1 / 2} \partial_{t} w, A^{1 / 2} \zeta\right) .
$$

For $\varepsilon$ sufficiently small,

$$
\mathcal{E}_{2}(t)-C \leq \Lambda_{2}(t) \leq 3 \mathcal{E}_{2}(t)+C .
$$

Besides, exploiting Lemma 5.3 and Lemma 5.4 with $\gamma=f(u)$ and $\varpi=1 / 20$, by standard computations we obtain

$$
\begin{aligned}
& \Phi(b)+4 \Psi(b)+\frac{7}{4} \int_{a}^{b}\|w(t)\|_{2}^{2} \mathrm{~d} t+2 \int_{a}^{b}\left\|\partial_{t} w(t)\right\|_{1}^{2} \mathrm{~d} t \\
& \leq \Phi(a)+4 \Psi(a)-4 M \int_{a}^{b} \int_{0}^{\infty}\left[\dot{\mu}_{t}(s)+\mu_{t}^{\prime}(s)\right]\left\|\zeta^{t}(s)\right\|_{2}^{2} \mathrm{~d} s \mathrm{~d} t \\
& \quad+C \int_{a}^{b} \kappa(t)\left\|\zeta^{t}\right\|_{\mathcal{M}_{t}^{1}}^{2} \mathrm{~d} t+C(b-a) .
\end{aligned}
$$

Adding this inequality with (8.2), and taking into account (8.3), we arrive at

$$
\Lambda_{2}(b)+2 \varepsilon \int_{a}^{b} \Lambda_{2}(t) \mathrm{d} t \leq \Lambda_{2}(a)+\frac{C}{\varepsilon}(b-a),
$$


up to fixing $\varepsilon$ sufficiently small. Hence, the Gronwall-type Lemma B.1 with $q_{1}=0$ and $q_{2}=C$ applies. Since $\Lambda_{2}(\tau)=0$, this gives (now $\varepsilon$ is fixed)

$$
\Lambda_{2}(t) \leq C, \quad \forall t \geq \tau
$$

and a further exploitation of (8.3) completes the argument.

Proof of Theorem 4.7. Up to taking $r>0$ sufficiently large, Lemma 8.1 tells that

$$
U_{1}(t, \tau) A_{\tau} \subset \mathbb{B}_{t}^{1}(r), \quad \forall t \geq \tau .
$$

Thus, for every $t \geq \tau$, we infer from the invariance of $\mathfrak{A}$ and (8.1) that

$$
\operatorname{dist}_{\mathcal{H}_{t}}\left(A_{t}, \mathbb{B}_{t}^{1}(r)\right)=\operatorname{dist}_{\mathcal{H}_{t}}\left(U(t, \tau) A_{\tau}, \mathbb{B}_{t}^{1}(r)\right) \leq C \mathrm{e}^{-\frac{\omega}{2}(t-\tau)} .
$$

Since the inequality holds for every $\tau \in \mathbb{R}$, letting $\tau \rightarrow-\infty$ we reach the conclusion

$$
\operatorname{dist}_{\mathcal{H}_{t}}\left(A_{t}, \mathbb{B}_{t}^{1}(r)\right)=0 \Rightarrow A_{t} \subset \mathbb{B}_{t}^{1}(r) \text {. }
$$

Accordingly, the $\mathcal{H}_{t}^{1}$-norm of $A_{t}$ is bounded by $r$ for every $t \in \mathbb{R}$.

\section{Recovering Kelvin-Voigt: Proof of Theorem 4.9}

By Remark 4.8, for every $n$ the function $w_{n}(\cdot)=u_{n}\left(\cdot+t_{n}\right)$ fulfills the equation

$$
\partial_{t t} w_{n}(t)+A w_{n}(t)+\int_{0}^{\infty} k_{t+t_{n}}(s) A \partial_{t} w_{n}(t-s) \mathrm{d} s+f\left(w_{n}(t)\right)=g .
$$

Besides, from the estimates of Theorem 4.7,

$$
w_{n} \text { is bounded in } L^{\infty}\left(\mathbb{R} ; H^{2}\right) \cap W^{1, \infty}\left(\mathbb{R} ; H^{1}\right) .
$$

Therefore, there exists

$$
\hat{u} \in L^{\infty}\left(\mathbb{R} ; H^{2}\right) \cap W^{1, \infty}\left(\mathbb{R} ; H^{1}\right)
$$

such that, up to a subsequence,

$$
\begin{aligned}
w_{n} \rightarrow \hat{u} & \text { weakly* in } L^{\infty}\left(\mathbb{R} ; \mathrm{H}^{2}\right), \\
\partial_{t} w_{n} \rightarrow \partial_{t} \hat{u} & \text { weakly* in } L^{\infty}\left(\mathbb{R} ; \mathrm{H}^{1}\right) .
\end{aligned}
$$

Since

$$
\lim _{n \rightarrow \infty} k_{t+t_{n}}=m \delta_{0}
$$

in the sense of (4.2), we deduce that, for every $T>0$,

$$
\sup _{t \in[-T, T]} \int_{0}^{\infty} k_{t+t_{n}}(s) \mathrm{d} s \leq 2 m
$$

for every $n$ sufficiently large (depending on $T$ ). Hence, exploiting (2.1) and the uniform estimate (9.2), by comparison in (9.1) we obtain, for all $n$ large,

$$
\sup _{t \in[-T, T]}\left\|\partial_{t t} w_{n}(t)\right\|_{-1} \leq C
$$

for some $C>0$ (which is actually independent of $T$ ), and we conclude that

$$
\partial_{t} w_{n} \text { is bounded in } L^{\infty}\left(-T, T ; \mathrm{H}^{1}\right) \cap W^{1, \infty}\left(-T, T ; \mathrm{H}^{-1}\right) .
$$

By the classical Simon-Aubin Theorem [31], we have the compact embeddings

$$
\begin{aligned}
L^{\infty}\left(-T, T ; \mathrm{H}^{2}\right) \cap W^{1, \infty}\left(-T, T ; \mathrm{H}^{1}\right) & \Subset \mathcal{C}\left([-T, T], \mathrm{H}^{1}\right), \\
L^{\infty}\left(-T, T ; \mathrm{H}^{1}\right) \cap W^{1, \infty}\left(-T, T ; \mathrm{H}^{-1}\right) & \Subset \mathcal{C}([-T, T], \mathrm{H}) .
\end{aligned}
$$


Accordingly, the strong convergence

$$
\left(w_{n}, \partial_{t} w_{n}\right) \rightarrow\left(\hat{u}, \partial_{t} \hat{u}\right) \quad \text { in } \mathcal{C}\left([-T, T], \mathrm{H}^{1} \times \mathrm{H}\right)
$$

holds (up to a subsequence), implying in particular (4.3). We are left to show that $\left(\hat{u}, \partial_{t} \hat{u}\right)$ solves the strongly damped wave equation, namely

$$
\partial_{t t} \hat{u}(t)+A \hat{u}(t)+A \partial_{t} \hat{u}(t)+f(\hat{u}(t))=g .
$$

Indeed, we are going to prove that the equality above is recovered when passing to the limit as $n \rightarrow \infty$ in (9.1), the only nonstandard convergence being

$$
\int_{0}^{\infty} k_{t+t_{n}}(s) A \partial_{t} w_{n}(t-s) \mathrm{d} s \rightarrow m A \partial_{t} \hat{u}(t)
$$

This follows if we can show that, for an arbitrarily fixed $t$,

$$
\mathfrak{I}_{n}(t)=\int_{0}^{\infty} k_{t+t_{n}}(s)\left\langle\partial_{t} w_{n}(t-s), A \phi\right\rangle \mathrm{d} s \rightarrow m\left\langle\partial_{t} \hat{u}(t), A \phi\right\rangle,
$$

for any sufficiently regular $\phi$ (say, $\phi \in \mathrm{H}^{2}$ ). To this end, let $\phi$ be fixed, and call for simplicity

$$
\varphi_{n}(\cdot)=\left\langle\partial_{t} w_{n}(\cdot), A \phi\right\rangle \quad \text { and } \quad \varphi(\cdot)=\left\langle\partial_{t} \hat{u}(\cdot), A \phi\right\rangle .
$$

Then, we write

$$
\mathfrak{I}_{n}(t)=\mathfrak{I}_{n}^{1}(t)+\mathfrak{I}_{n}^{2}(t)+\mathfrak{I}_{n}^{3}(t)
$$

where

$$
\begin{aligned}
& \mathfrak{I}_{n}^{1}(t)=\int_{0}^{1} k_{t+t_{n}}(s) \varphi(t-s) \mathrm{d} s \\
& \mathfrak{I}_{n}^{2}(t)=\int_{0}^{1} k_{t+t_{n}}(s)\left[\varphi_{n}(t-s)-\varphi(t-s)\right] \mathrm{d} s, \\
& \mathfrak{I}_{n}^{3}(t)=\int_{1}^{\infty} k_{t+t_{n}}(s) \varphi_{n}(t-s) \mathrm{d} s .
\end{aligned}
$$

Since $\varphi_{n} \in L^{\infty}(\mathbb{R})$ uniformly with respect to $n$, and $\varphi_{n} \rightarrow \varphi$ in $\mathcal{C}(I)$ for every closed interval $I$, it is apparent from (4.2) that

$$
\left|\mathfrak{I}_{n}^{2}(t)+\mathfrak{I}_{n}^{3}(t)\right| \leq\left\|\varphi_{n}-\varphi\right\|_{\mathcal{C}([t-1, t])} \int_{0}^{1} k_{t+t_{n}}(s) \mathrm{d} s+\left\|\varphi_{n}\right\|_{L^{\infty}(\mathbb{R})} \int_{1}^{\infty} k_{t+t_{n}}(s) \mathrm{d} s \rightarrow 0
$$

while

$$
\mathfrak{I}_{n}^{1}(t) \rightarrow m \varphi(t)
$$

proving the required convergence.

\section{Two Memory Kernels of Physical Interest}

In this final section we discuss two concrete examples of time-dependent memory kernels arising in the physical applications, already introduced in [4]. 
I. The rescaled kernel. Let $\mu \in \mathcal{C}^{1}\left(\mathbb{R}^{+}\right) \cap L^{1}\left(\mathbb{R}^{+}\right)$be a (nonnull and nonnegative) nonincreasing function with $\mu(0)<\infty$. Given a bounded positive function $\varepsilon \in \mathcal{C}^{1}(\mathbb{R})$ satisfying

$$
\dot{\varepsilon}(t) \leq 0, \quad \forall t \in \mathbb{R}
$$

we define the time-dependent rescaled kernel

$$
\mu_{t}(s)=\frac{1}{[\varepsilon(t)]^{2}} \mu\left(\frac{s}{\varepsilon(t)}\right) .
$$

According to (1.5), the corresponding integrated memory kernel reads

$$
k_{t}(s)=\frac{1}{\varepsilon(t)} k\left(\frac{s}{\varepsilon(t)}\right) \quad \text { where } \quad k(s)=\int_{s}^{\infty} \mu(y) \mathrm{d} y .
$$

In particular, assuming $k$ summable with total mass $m>0$, the most interesting situation is when $\varepsilon(t) \rightarrow 0$ as $t \rightarrow \infty$. In which case, we recover the distributional convergence $k_{t} \rightarrow m \delta_{0}$ to (a multiple of) the Dirac mass at zero. As shown in [4], this $\mu_{t}$ complies with (M1)-(M3). Here, we make two further assumptions: there exists $\varrho>0$ such that

$$
\mu^{\prime}(s)+\varrho \mu(s) \leq 0, \quad \forall s>0,
$$

and

$$
\inf _{t \in \mathbb{R}} \dot{\varepsilon}(t)>-\frac{\varrho}{2}
$$

Example. For instance, a possible choice is the exponential kernel $\mu(s)=\mathrm{e}^{-s}$ and

$$
\varepsilon(t)=c\left[\frac{\pi}{2}-\arctan (t)\right], \quad 0<c<\frac{1}{2} .
$$

Our aim is showing that, under (10.1)-(10.2), the rescaled kernel $\mu_{t}$ satisfies (M4)(M8) as well. To this end, we recall that the derivatives of $\mu_{t}$ read

$$
\mu_{t}^{\prime}(s)=\frac{1}{[\varepsilon(t)]^{3}} \mu^{\prime}\left(\frac{s}{\varepsilon(t)}\right) \quad \text { and } \quad \dot{\mu}_{t}(s)=-\frac{\dot{\varepsilon}(t)}{\varepsilon(t)}\left[2 \mu_{t}(s)+s \mu_{t}^{\prime}(s)\right] \text {. }
$$

Besides,

$$
\kappa(t)=\int_{0}^{\infty} \mu_{t}(s) \mathrm{d} s=\frac{\kappa}{\varepsilon(t)},
$$

being $\kappa=\int_{0}^{\infty} \mu(s) \mathrm{d} s$ the total mass of $\mu$.

- Verifying (M4). On account of (10.2), choose $\delta>0$ small enough that

$$
\delta \kappa-2 \dot{\varepsilon}(t) \leq \varrho .
$$

Then, for every $t \in \mathbb{R}$ and $s>0$,

$$
\begin{aligned}
\dot{\mu}_{t}(s)+\mu_{t}^{\prime}(s)+\delta \kappa(t) \mu_{t}(s) & =\left[1-\frac{s \dot{\varepsilon}(t)}{\varepsilon(t)}\right] \mu_{t}^{\prime}(s)+[\delta \kappa-2 \dot{\varepsilon}(t)] \frac{\mu_{t}(s)}{\varepsilon(t)} \\
& \leq \mu_{t}^{\prime}(s)+\frac{\varrho}{\varepsilon(t)} \mu_{t}(s) \\
& =\frac{1}{[\varepsilon(t)]^{3}}\left[\mu^{\prime}\left(\frac{s}{\varepsilon(t)}\right)+\varrho \mu\left(\frac{s}{\varepsilon(t)}\right)\right] \leq 0
\end{aligned}
$$

The latter inequality follows from (10.1). 
- Verifying (M5). Simply observe that

$$
\inf _{t \in \mathbb{R}} \kappa(t)=\lim _{t \rightarrow-\infty} \frac{\kappa}{\varepsilon(t)}>0 .
$$

- Verifying (M6). In the light of (M1), an integration by parts readily gives

$$
\int_{0}^{\infty} s \mu_{t}^{\prime}(s) \mathrm{d} s=-\kappa(t) .
$$

Hence, recalling that $\dot{\varepsilon} \leq 0$ and $\mu_{t}^{\prime} \leq 0$,

$$
\int_{0}^{\infty}\left|\dot{\mu}_{t}(s)\right| \mathrm{d} s=-\frac{\dot{\varepsilon}(t)}{\varepsilon(t)} \int_{0}^{\infty}\left|2 \mu_{t}(s)+s \mu_{t}^{\prime}(s)\right| \mathrm{d} s \leq-\frac{3 \kappa(t) \dot{\varepsilon}(t)}{\varepsilon(t)}=-\frac{3 \kappa \dot{\varepsilon}(t)}{[\varepsilon(t)]^{2}},
$$

and exploiting (10.2),

$$
\sup _{t \in \mathbb{R}} \frac{1}{[\kappa(t)]^{2}} \int_{0}^{\infty}\left|\dot{\mu}_{t}(s)\right| \mathrm{d} s \leq-\inf _{t \in \mathbb{R}} \frac{3 \dot{\varepsilon}(t)}{\kappa}<\frac{3 \varrho}{2 \kappa} .
$$

- Verifying (M7). By direct calculations,

$$
\frac{\mu_{t}(0)}{[\kappa(t)]^{2}}=\frac{\mu(0)}{\kappa^{2}}, \quad \forall t \in \mathbb{R} .
$$

- Verifying (M8). For $t \in[a, b]$ and $\nu>0$, we have

$$
\int_{\nu}^{1 / \nu} \mu_{t}(s) \mathrm{d} s=\left(\frac{1}{\kappa} \int_{\nu / \varepsilon(t)}^{1 / \nu \varepsilon(t)} \mu(s) \mathrm{d} s\right) \kappa(t) \geq\left(\frac{1}{\kappa} \int_{\nu / \varepsilon(a)}^{1 / \nu \varepsilon(b)} \mu(s) \mathrm{d} s\right) \kappa(t) .
$$

When $\nu \rightarrow 0$, the right-hand side converges to $\kappa(t)$.

II. The rheological kernel. The second example of time-dependent memory kernel comes from a physical derivation of equation (1.4) via a rheological model for aging materials (see [4, Appendix] for details). It has the form

$$
\mu_{t}(s)=\frac{1}{\varrho \gamma} \mathrm{K}_{0}(t) \mathrm{K}_{0}(t-s) \mathrm{e}^{-\frac{1}{\gamma} \int_{0}^{s} \mathrm{~K}_{0}(t-y) \mathrm{d} y},
$$

where $\varrho, \gamma$ are positive constants and $\mathrm{K}_{0} \in \mathcal{C}^{1}(\mathbb{R})$ is a nondecreasing function such that

$$
\lim _{t \rightarrow-\infty} \mathrm{K}_{0}(t)=\beta>0 \text {. }
$$

The corresponding integrated memory kernel given by (1.5) reads

$$
k_{t}(s)=\frac{1}{\varrho} \mathrm{K}_{0}(t) \mathrm{e}^{-\frac{1}{\gamma} \int_{0}^{s} \mathrm{~K}_{0}(t-y) \mathrm{d} y} .
$$

Here, the interesting situation is when $\mathrm{K}_{0}(t) \rightarrow \infty$ as $t \rightarrow \infty$, translating the physical assumption that the spring in the Maxwell element of the rheological model becomes completely rigid in the longtime. In which case, quite remarkably, we have the distributional convergence (see [4])

$$
k_{t} \rightarrow \frac{\gamma}{\varrho} \delta_{0} \quad \text { as } t \rightarrow \infty .
$$


In the same paper, the rheological kernel $\mu_{t}$ is shown to satisfy (M1)-(M3). In order to verify the remaining axioms (M4)-(M8), a further assumptions is needed: there exists a positive constant $\mathrm{M}<\beta / \gamma$ such that

$$
\dot{\mathrm{K}}_{0}(t) \leq \mathrm{MK}_{0}(t), \quad \forall t \in \mathbb{R}
$$

Loosely speaking, (10.4) prevents $\mathrm{K}_{0}$ to grow "too fast". On the other hand, $\mathrm{K}_{0}$ can even be an exponential.

Example. The exponential function

$$
\mathrm{K}_{0}(t)=1+\mathrm{e}^{\alpha t}
$$

fulfills our hypotheses, provided that $0<\alpha<1 / \gamma$.

First, let us write explicitly the derivatives of $\mu_{t}$. Namely,

$$
\mu_{t}^{\prime}(s)=-\frac{\mathrm{K}_{0}(t)}{\varrho \gamma}\left[\dot{\mathrm{K}}_{0}(t-s)+\frac{1}{\gamma}\left[\mathrm{K}_{0}(t-s)\right]^{2}\right] \mathrm{e}^{-\frac{1}{\gamma} \int_{0}^{s} \mathrm{~K}_{0}(t-y) \mathrm{d} y}
$$

and

$$
\begin{aligned}
\dot{\mu}_{t}(s)=\frac{1}{\varrho \gamma}\left[\dot{\mathrm{K}}_{0}(t) \mathrm{K}_{0}(t-s)+\mathrm{K}_{0}(t) \dot{\mathrm{K}}_{0}(t-s)\right. \\
\left.\quad-\frac{1}{\gamma}\left[\mathrm{K}_{0}(t)\right]^{2} \mathrm{~K}_{0}(t-s)+\frac{1}{\gamma} \mathrm{K}_{0}(t)\left[\mathrm{K}_{0}(t-s)\right]^{2}\right] \mathrm{e}^{-\frac{1}{\gamma} \int_{0}^{s} \mathrm{~K}_{0}(t-y) \mathrm{d} y} .
\end{aligned}
$$

Besides,

$$
\kappa(t)=\int_{0}^{\infty} \mu_{t}(s) \mathrm{d} s=\frac{\mathrm{K}_{0}(t)}{\varrho} .
$$

- Verifying (M4). By virtue of (10.4), let $0<\delta \leq \varrho / \gamma$ to be properly chosen. By explicit calculations,

$$
\dot{\mu}_{t}(s)+\mu_{t}^{\prime}(s)+\delta \kappa(t) \mu_{t}(s)=\frac{\mathrm{K}_{0}(t-s)}{\varrho^{2} \gamma^{2}} \mathrm{~F}(t) \mathrm{e}^{-\frac{1}{\gamma} \int_{0}^{s} \mathrm{~K}_{0}(t-r) \mathrm{d} r},
$$

where

$$
\mathrm{F}(t)=\varrho \gamma \dot{\mathrm{K}}_{0}(t)+(\delta \gamma-\varrho)\left[\mathrm{K}_{0}(t)\right]^{2} .
$$

The claim amounts to showing that $\mathrm{F} \leq 0$. Indeed, since $\mathrm{K}_{0} \geq \beta$ and $\delta \gamma-\varrho \leq 0$, we infer from (10.4) that

$$
\mathrm{F}(t) \leq[\varrho \gamma \mathrm{M}+(\delta \gamma-\varrho) \beta] \mathrm{K}_{0}(t) \leq 0,
$$

as long as we fix $\delta>0$ small enough that

$$
\delta \leq \frac{\varrho(\beta-\gamma \mathrm{M})}{\gamma \beta}
$$

This is possible because $\beta-\gamma \mathrm{M}>0$.

- Verifying (M5). Due to (10.3) and the fact that $\dot{\mathrm{K}}_{0} \geq 0$,

$$
\inf _{t \in \mathbb{R}} \kappa(t)=\frac{1}{\varrho} \inf _{t \in \mathbb{R}} \mathrm{K}_{0}(t)=\frac{\beta}{\varrho}>0 .
$$

- Verifying (M6). Since $\dot{\mathrm{K}}_{0} \geq 0$ and (10.3)-(10.4) hold,

$$
\left|\dot{\mu}_{t}(s)\right| \leq \frac{2\left[\mathrm{~K}_{0}(t)\right]^{2}}{\varrho \gamma}\left(\frac{\mathrm{M}}{\beta}+\frac{1}{\gamma}\right) \mathrm{K}_{0}(t-s) \mathrm{e}^{-\frac{1}{\gamma} \int_{0}^{s} \mathrm{~K}_{0}(t-r) \mathrm{d} r} .
$$


Moreover, as the positive function $\mathrm{K}_{0}$ remains away from zero,

$$
\int_{0}^{\infty} \mathrm{K}_{0}(t-s) \mathrm{e}^{-\frac{1}{\gamma} \int_{0}^{s} \mathrm{~K}_{0}(t-r) \mathrm{d} r} \mathrm{~d} s=\gamma-\gamma \lim _{s \rightarrow \infty} \mathrm{e}^{-\frac{1}{\gamma} \int_{0}^{s} \mathrm{~K}_{0}(t-r) \mathrm{d} r} \mathrm{~d} s=\gamma .
$$

Accordingly,

Hence,

$$
\int_{0}^{\infty}\left|\dot{\mu}_{t}(s)\right| \mathrm{d} s \leq \frac{2\left[\mathrm{~K}_{0}(t)\right]^{2}}{\varrho}\left(\frac{\mathrm{M}}{\beta}+\frac{1}{\gamma}\right) .
$$

$$
\sup _{t \in \mathbb{R}} \frac{1}{[\kappa(t)]^{2}} \int_{0}^{\infty}\left|\dot{\mu}_{t}(s)\right| \mathrm{d} s \leq 2 \varrho\left(\frac{\mathrm{M}}{\beta}+\frac{1}{\gamma}\right) .
$$

- Verifying (M7). By the very definition of $\mu_{t}$,

$$
\frac{\mu_{t}(0)}{[\kappa(t)]^{2}}=\frac{\varrho}{\gamma}, \quad \forall t \in \mathbb{R}
$$

- Verifying (M8). Recalling the form of $k_{t}(s)$, thanks to (10.3) we easily get

$$
\int_{\nu}^{1 / \nu} \mu_{t}(s) \mathrm{d} s=\left[\mathrm{e}^{-\frac{1}{\gamma} \int_{0}^{\nu} \mathrm{K}_{0}(t-y) \mathrm{d} y}-\mathrm{e}^{-\frac{1}{\gamma} \int_{0}^{1 / \nu} \mathrm{K}_{0}(t-y) \mathrm{d} y}\right] \frac{\mathrm{K}_{0}(t)}{\varrho} \rightarrow \kappa(t)
$$

as $\nu \rightarrow 0$, uniformly as $t \in[a, b]$.

\section{Appendix A}

Proof of Lemma 5.3

Let $b>a \geq \tau$ be arbitrarily fixed. In what follows $\left(p, \partial_{t} p, \psi\right)$ will be a solution to problem (5.1)-(5.3), as regular as needed. In particular, we assume $\psi_{\tau} \in \mathfrak{D}\left(\mathbb{T}_{\tau}\right) \cap \mathcal{C}^{1}\left(\mathbb{R}^{+}, \mathrm{H}^{1}\right)$ and $p \in W^{2, \infty}\left(a, b ; \mathrm{H}^{1}\right)$. In addition, the derivative $\psi_{\tau}^{\prime}$ of the initial datum $\psi_{\tau}$ is required to fulfill $\psi_{\tau}^{\prime} \in L^{\infty}\left(0, s_{0} ; \mathrm{H}\right)$ for every $s_{0}>0$. In fact, since we are always working in a Galerkin regularization scheme, this is no loss of generality. According to [4, Section 5], we can differentiate (5.2) with respect to $s$ in the weak sense, so obtaining

$$
\partial_{s} \psi^{t}(s)= \begin{cases}\partial_{t} p(t-s), & s \leq t-\tau, \\ \psi_{\tau}^{\prime}(s-t+\tau), & s>t-\tau .\end{cases}
$$

Besides, $\psi^{t}$ belongs to $\mathfrak{D}\left(\mathbb{T}_{\tau}\right)$ for every $t \geq \tau$ and satisfies the differential equality in $\mathcal{M}_{\tau}$

$$
\partial_{t} \psi^{t}=\mathbb{T}_{\tau} \psi^{t}+\partial_{t} p(t)
$$

The proof of Lemma 5.3 will be carried out in a number of steps. The generic constant $C>0$ appearing in the next lines is understood to be independent of the interval $[a, b]$. Instead, we will denote by $D>0$ a generic constant depending explicitly on $[a, b]$.

I. The approximating functional. We introduce a family of approximate memory kernels with compact support in $\mathbb{R}^{+}$. For $\varepsilon>0$ small, we define the cut-off function

$$
\phi_{\varepsilon}(s)= \begin{cases}0 & \text { if } 0 \leq s<\varepsilon \\ s / \varepsilon-1 & \text { if } \varepsilon \leq s<2 \varepsilon \\ 1 & \text { if } 2 \varepsilon \leq s \leq 1 / \varepsilon \\ 2-\varepsilon s & \text { if } 1 / \varepsilon \leq s<2 / \varepsilon \\ 0 & \text { if } 2 / \varepsilon \leq s\end{cases}
$$


along with the approximate kernel

$$
\mu_{t}^{\varepsilon}(s)=\phi_{\varepsilon}(s) \mu_{t}(s) \leq \mu_{t}(s) .
$$

Setting

$$
\kappa_{\varepsilon}(t)=\int_{0}^{\infty} \mu_{t}^{\varepsilon}(s) \mathrm{d} s \leq \kappa(t)
$$

we introduce the functional

$$
\Psi_{\varepsilon}(t)=-\frac{2}{\kappa_{\varepsilon}(t)} \int_{0}^{\infty} \mu_{t}^{\varepsilon}(s)\left\langle\psi^{t}(s), \partial_{t} p(t)\right\rangle \mathrm{d} s,
$$

and we define

$$
R_{\varepsilon}(t)=\left[\frac{\kappa(t)}{\kappa_{\varepsilon}(t)}\right]^{2} \geq 1 .
$$

Owing to (M8), for all $\varepsilon$ sufficiently small (depending on $a$ and $b$ ), we have

$$
\sup _{t \in[a, b]} R_{\varepsilon}(t) \leq 4
$$

The time-derivative of $\Psi_{\varepsilon}$ is given by

$$
\frac{\mathrm{d}}{\mathrm{d} t} \Psi_{\varepsilon}(t)=\mathfrak{I}_{1}(t)+\mathfrak{I}_{2}(t)
$$

where $^{8}$

$$
\begin{aligned}
& \mathfrak{I}_{1}(t)=-2 \frac{\mathrm{d}}{\mathrm{d} t}\left[\frac{1}{\kappa_{\varepsilon}(t)}\right] \int_{0}^{\infty} \mu_{t}^{\varepsilon}(s)\left\langle\psi(s), \partial_{t} p\right\rangle \mathrm{d} s, \\
& \mathfrak{I}_{2}(t)=-\frac{2}{\kappa_{\varepsilon}(t)} \frac{\mathrm{d}}{\mathrm{d} t} \int_{0}^{\infty} \mu_{t}^{\varepsilon}(s)\left\langle\psi(s), \partial_{t} p\right\rangle \mathrm{d} s .
\end{aligned}
$$

II. Estimating $\mathfrak{I}_{1}$. We first observe that

$$
\frac{\mathrm{d}}{\mathrm{d} t}\left[\frac{1}{\kappa_{\varepsilon}(t)}\right]=-\frac{1}{\left[\kappa_{\varepsilon}(t)\right]^{2}} \frac{\mathrm{d}}{\mathrm{d} t} \int_{0}^{\infty} \mu_{t}^{\varepsilon}(s)=-\frac{1}{\left[\kappa_{\varepsilon}(t)\right]^{2}} \int_{0}^{\infty} \dot{\mu}_{t}^{\varepsilon}(s) .
$$

Passing the derivative through the integral sign is allowed by a classical result. Indeed, we know from (M1) and (M3) that

- the map $s \mapsto \mu_{t}^{\varepsilon}(s)$ is summable on $\mathbb{R}^{+}$for every fixed $t$;

- for almost every fixed $s>0$, the map $t \mapsto \mu_{t}^{\varepsilon}(s)$ is differentiable for all $t \in \mathbb{R}$;

- for every fixed closed interval $I \subset \mathbb{R}$ and almost every $t \in I$,

$$
\left|\dot{\mu}_{t}^{\varepsilon}(s)\right| \leq\|\dot{\mu}\|_{L^{\infty}(I \times[\varepsilon, 1 / \varepsilon])} \chi_{[\varepsilon, 1 / \varepsilon]}(s) \in L^{1}\left(\mathbb{R}^{+}\right) .
$$

Accordingly, thanks to (M6) and (A.3),

$$
\begin{aligned}
\mathfrak{I}_{1}(t) & \leq 2 R_{\varepsilon}(t)\left\|\partial_{t} p\right\| \frac{1}{[\kappa(t)]^{2}} \int_{0}^{\infty}\left|\dot{\mu}_{t}(s)\right| \mathrm{d} s \int_{0}^{\infty} \mu_{t}(s)\|\psi(s)\| \mathrm{d} s \\
& \leq \frac{1}{2} R_{\varepsilon}(t)\left\|\partial_{t} p\right\|^{2}+C \kappa(t)\|\psi\|_{\mathcal{M}_{t}}^{2} .
\end{aligned}
$$

\footnotetext{
${ }^{8}$ We omit for simplicity of notation the dependence on $t$ of the variables $p$ and $\psi$.
} 
III. Estimating $\mathfrak{I}_{2}$. Passing again the derivative within the integral (arguing as before), we write

$$
\mathfrak{I}_{2}(t)=\mathfrak{F}(t)-2\left\|\partial_{t} p\right\|^{2}-\frac{2 \sqrt{R_{\varepsilon}(t)}}{\kappa(t)} \int_{0}^{\infty} \mu_{t}^{\varepsilon}(s)\left\langle\psi(s), \partial_{t t} p\right\rangle \mathrm{d} s,
$$

where we set

$$
\mathfrak{F}(t)=2\left\|\partial_{t} p\right\|^{2}-\frac{2}{\kappa_{\varepsilon}(t)}\left[\int_{0}^{\infty} \dot{\mu}_{t}^{\varepsilon}(s)\left\langle\psi(s), \partial_{t} p\right\rangle \mathrm{d} s+\int_{0}^{\infty} \mu_{t}^{\varepsilon}(s)\left\langle\partial_{t} \psi(s), \partial_{t} p\right\rangle \mathrm{d} s\right] .
$$

We preliminarily observe that, exploiting (A.2),

$$
\begin{aligned}
\int_{0}^{\infty} \mu_{t}^{\varepsilon}(s)\left\langle\partial_{t} \psi(s), \partial_{t} p\right\rangle \mathrm{d} s & =-\int_{0}^{\infty} \mu_{t}^{\varepsilon}(s)\left\langle\partial_{s} \psi(s), \partial_{t} p\right\rangle \mathrm{d} s+\kappa_{\varepsilon}(t)\left\|\partial_{t} p\right\|^{2} \\
& =\int_{0}^{\infty}\left(\mu_{t}^{\varepsilon}\right)^{\prime}(s)\left\langle\psi(s), \partial_{t} p\right\rangle \mathrm{d} s+\kappa_{\varepsilon}(t)\left\|\partial_{t} p\right\|^{2}
\end{aligned}
$$

The latter equality follows from an integration by parts, where the boundary terms are easily seen to vanish. Hence,

$$
\mathfrak{F}(t)=-\frac{2}{\kappa_{\varepsilon}(t)} \int_{0}^{\infty}\left[\dot{\mu}_{t}^{\varepsilon}(s)+\left(\mu_{t}^{\varepsilon}\right)^{\prime}(s)\right]\left\langle\psi(s), \partial_{t} p\right\rangle \mathrm{d} s .
$$

At this point, we further decompose $\mathfrak{F}$ into the sum

$$
\mathfrak{F}(t)=\mathfrak{F}_{1}(t)+\mathfrak{F}_{2}(t),
$$

where

$$
\begin{aligned}
& \mathfrak{F}_{1}(t)=-\frac{2}{\kappa_{\varepsilon}(t)} \int_{0}^{\infty} \phi_{\varepsilon}(s)\left[\dot{\mu}_{t}(s)+\mu_{t}^{\prime}(s)\right]\left\langle\psi(s), \partial_{t} p\right\rangle \mathrm{d} s \\
& \mathfrak{F}_{2}(t)=-\frac{2}{\kappa_{\varepsilon}(t)} \int_{0}^{\infty} \phi_{\varepsilon}^{\prime}(s) \mu_{t}(s)\left\langle\psi(s), \partial_{t} p\right\rangle \mathrm{d} s .
\end{aligned}
$$

We estimate these two terms separately.

- Since $\dot{\mu}+\mu^{\prime} \leq 0$,

$$
\begin{aligned}
\mathfrak{F}_{1}(t) & \leq 2 \sqrt{R_{\varepsilon}(t)}\left\|\partial_{t} p\right\| \frac{1}{\kappa(t)} \int_{0}^{\infty}-\left[\dot{\mu}_{t}(s)+\mu_{t}^{\prime}(s)\right]\|\psi(s)\| \mathrm{d} s \\
& \leq \frac{1}{2} R_{\varepsilon}(t)\left\|\partial_{t} p\right\|^{2}+\frac{C}{[\kappa(t)]^{2}}\left[\int_{0}^{\infty}-\left[\dot{\mu}_{t}(s)+\mu_{t}^{\prime}(s)\right]\|\psi(s)\|_{1} \mathrm{~d} s\right]^{2} .
\end{aligned}
$$

Moreover, owing to (M6)-(M7),

$$
\begin{aligned}
& \frac{C}{[\kappa(t)]^{2}}\left[\int_{0}^{\infty}-\left[\dot{\mu}_{t}(s)+\mu_{t}^{\prime}(s)\right]\|\psi(s)\|_{1} \mathrm{~d} s\right]^{2} \\
& \quad \leq \frac{C}{[\kappa(t)]^{2}} \int_{0}^{\infty}-\left[\dot{\mu}_{t}(s)+\mu_{t}^{\prime}(s)\right] \mathrm{d} s \int_{0}^{\infty}-\left[\dot{\mu}_{t}(s)+\mu_{t}^{\prime}(s)\right]\|\psi(s)\|_{1}^{2} \mathrm{~d} s \\
& \quad \leq C\left[\frac{1}{[\kappa(t)]^{2}} \int_{0}^{\infty}\left|\dot{\mu}_{t}(s)\right| \mathrm{d} s+\frac{\mu_{t}(0)}{[\kappa(t)]^{2}}\right] \int_{0}^{\infty}-\left[\dot{\mu}_{t}(s)+\mu_{t}^{\prime}(s)\right]\|\psi(s)\|_{1}^{2} \mathrm{~d} s \\
& \quad \leq-M \int_{0}^{\infty}\left[\dot{\mu}_{t}(s)+\mu_{t}^{\prime}(s)\right]\|\psi(s)\|_{1}^{2} \mathrm{~d} s
\end{aligned}
$$


for some $M>0$, independent of $[a, b]$. Therefore, we end up with the estimate

$$
\mathfrak{F}_{1}(t) \leq \frac{1}{2} R_{\varepsilon}(t)\left\|\partial_{t} p\right\|^{2}-M \int_{0}^{\infty}\left[\dot{\mu}_{t}(s)+\mu_{t}^{\prime}(s)\right]\|\psi(s)\|_{1}^{2} \mathrm{~d} s .
$$

- Since

$$
\left|\phi_{\varepsilon}^{\prime}(s)\right| \leq \frac{1}{\varepsilon} \chi_{[\varepsilon, 2 \varepsilon]}(s)+\varepsilon,
$$

making use of (M5), (A.3) and the control $\partial_{t} p \in L^{\infty}(a, b ; \mathrm{H})$, we get

$$
\mathfrak{F}_{2}(t) \leq D \int_{0}^{\infty} r_{\varepsilon}(t, s) \mathrm{d} s, \quad \forall t \in[a, b],
$$

having set

$$
r_{\varepsilon}(t, s)=\left(\frac{1}{\varepsilon} \chi_{[\varepsilon, 2 \varepsilon]}(s)+\varepsilon\right) \mu_{t}(s)\left\|\psi^{t}(s)\right\| .
$$

Collecting the two inequalities, we conclude that

$$
\mathfrak{F}(t) \leq \frac{1}{2} R_{\varepsilon}(t)\left\|\partial_{t} p\right\|^{2}-M \int_{0}^{\infty}\left[\dot{\mu}_{t}(s)+\mu_{t}^{\prime}(s)\right]\|\psi(s)\|_{1}^{2} \mathrm{~d} s+D \int_{0}^{\infty} r_{\varepsilon}(t, s) \mathrm{d} s .
$$

In turn, this yields

$$
\begin{aligned}
\mathfrak{I}_{2}(t) \leq & -\left(2-\frac{1}{2} R_{\varepsilon}(t)\right)\left\|\partial_{t} p\right\|^{2}-M \int_{0}^{\infty}\left[\dot{\mu}_{t}(s)+\mu_{t}^{\prime}(s)\right]\|\psi(s)\|_{1}^{2} \mathrm{~d} s \\
& +D \int_{0}^{\infty} r_{\varepsilon}(t, s) \mathrm{d} s-\frac{2 \sqrt{R_{\varepsilon}(t)}}{\kappa(t)} \int_{0}^{\infty} \mu_{t}^{\varepsilon}(s)\left\langle\psi(s), \partial_{t t} p\right\rangle \mathrm{d} s .
\end{aligned}
$$

IV. The integral inequality. Plugging (A.5) and (A.7) into (A.4), and integrating on $[a, b]$, we are led to

$$
\begin{aligned}
\Psi_{\varepsilon}(b) & +\int_{a}^{b}\left[2-R_{\varepsilon}(t)\right]\left\|\partial_{t} p(t)\right\|^{2} \mathrm{~d} t \\
\leq & \Psi_{\varepsilon}(a)-M \int_{a}^{b} \int_{0}^{\infty}\left[\dot{\mu}_{t}(s)+\mu_{t}^{\prime}(s)\right]\left\|\psi^{t}(s)\right\|_{1}^{2} \mathrm{~d} s \mathrm{~d} t+C \int_{a}^{b} \kappa(t)\left\|\psi^{t}\right\|_{\mathcal{M}_{t}}^{2} \mathrm{~d} t \\
& +D \int_{a}^{b} \int_{0}^{\infty} r_{\varepsilon}(t, s) \mathrm{d} s \mathrm{~d} t-\int_{a}^{b} \frac{2 \sqrt{R_{\varepsilon}(t)}}{\kappa(t)} \int_{0}^{\infty} \mu_{t}^{\varepsilon}(s)\left\langle\psi^{t}(s), \partial_{t t} p(t)\right\rangle \mathrm{d} s \mathrm{~d} t .
\end{aligned}
$$

The next step is to let $\varepsilon \rightarrow 0$, so to obtain

$$
\begin{aligned}
\Psi(b) & +\int_{a}^{b}\left\|\partial_{t} p(t)\right\|^{2} \mathrm{~d} t \\
\leq & \Psi(a)-M \int_{a}^{b} \int_{0}^{\infty}\left[\dot{\mu}_{t}(s)+\mu_{t}^{\prime}(s)\right]\left\|\psi^{t}(s)\right\|_{1}^{2} \mathrm{~d} s \mathrm{~d} t+C \int_{a}^{b} \kappa(t)\left\|\psi^{t}\right\|_{\mathcal{M}_{t}}^{2} \mathrm{~d} t \\
& -\int_{a}^{b} \frac{2}{\kappa(t)} \int_{0}^{\infty} \mu_{t}(s)\left\langle\psi^{t}(s), \partial_{t t} p(t)\right\rangle \mathrm{d} s \mathrm{~d} t .
\end{aligned}
$$

This will follow from a repeated use of the Dominated Convergence Theorem (DCT).

- We first show that $\Psi_{\varepsilon}(t) \rightarrow \Psi(t)$ as $\varepsilon \rightarrow 0$. Indeed, for any fixed $t \in[a, b]$, it is apparent that $\kappa_{\varepsilon}(t) \rightarrow \kappa(t)$, and

$$
\mu_{t}^{\varepsilon}(s)\left\langle\psi^{t}(s), \partial_{t} p(t)\right\rangle \rightarrow \mu_{t}(s)\left\langle\psi^{t}(s), \partial_{t} p(t)\right\rangle, \quad \forall s>0 .
$$


Since

$$
\mu_{t}^{\varepsilon}(s)\left|\left\langle\psi^{t}(s), \partial_{t} p(t)\right\rangle\right| \leq \mu_{t}(s)\left\|\psi^{t}(s)\right\|\left\|\partial_{t} p(t)\right\| \in L^{1}\left(\mathbb{R}^{+}\right),
$$

the claim follows from the DCT.

- For every fixed $t \in[a, b]$, we have the convergence $R_{\varepsilon}(t) \rightarrow 1$. Keeping in mind (A.3), from the DCT we easily infer that

$$
\int_{a}^{b}\left[2-R_{\varepsilon}(t)\right]\left\|\partial_{t} p(t)\right\|^{2} \mathrm{~d} t \rightarrow \int_{a}^{b}\left\|\partial_{t} p(t)\right\|^{2} \mathrm{~d} t
$$

- Similarly, by applying the DCT on $[a, b] \times \mathbb{R}^{+}$, we prove that

$$
\int_{a}^{b} \frac{2 \sqrt{R_{\varepsilon}(t)}}{\kappa(t)} \int_{0}^{\infty} \mu_{t}^{\varepsilon}(s)\left\langle\psi^{t}(s), \partial_{t t} p(t)\right\rangle \mathrm{d} s \mathrm{~d} t \rightarrow \int_{a}^{b} \frac{2}{\kappa(t)} \int_{0}^{\infty} \mu_{t}(s)\left\langle\psi^{t}(s), \partial_{t t} p(t)\right\rangle \mathrm{d} s \mathrm{~d} t .
$$

Indeed, on account of (M5), (A.3) together with the control $\partial_{t t} p \in L^{\infty}(a, b ; \mathrm{H})$,

$$
\left|\frac{2 \sqrt{R_{\varepsilon}(t)}}{\kappa(t)} \mu_{t}^{\varepsilon}(s)\left\langle\psi^{t}(s), \partial_{t t} p(t)\right\rangle\right| \leq D \mu_{t}(s)\left\|\psi^{t}(s)\right\|_{1},
$$

and

$$
\int_{0}^{\infty} \mu_{t}(s)\left\|\psi^{t}(s)\right\|_{1} \mathrm{~d} s \leq \sqrt{\kappa(t)}\left\|\psi^{t}\right\|_{\mathcal{M}_{t}} \in L^{1}(a, b)
$$

due to Remark 5.2.

- We are left to show that

$$
\int_{a}^{b} \int_{0}^{\infty} r_{\varepsilon}(t, s) \mathrm{d} s \mathrm{~d} t \rightarrow 0
$$

Recalling (A.6), we immediately draw the pointwise convergence

$$
r_{\varepsilon}(t, s) \rightarrow 0, \quad \forall(t, s) \in[a, b] \times \mathbb{R}^{+} .
$$

By virtue of (A.1) and the hypotheses on $p$ and $\psi_{\tau}$, we learn that

$$
\left\|\partial_{s} \psi^{t}\right\|_{L^{\infty}(0,2 ; \mathrm{H})}^{2} \leq D, \quad \forall t \in[a, b] .
$$

Therefore, as $\psi^{t} \in \mathfrak{D}\left(\mathbb{T}_{\tau}\right)$,

$$
\left\|\psi^{t}(s)\right\| \leq \int_{0}^{s}\left\|\partial_{s} \psi^{t}(y)\right\| \mathrm{d} y \leq D s, \quad \forall s \in(0,2] .
$$

This allows us to obtain the estimate in $[a, b] \times \mathbb{R}^{+}$

$$
r_{\varepsilon}(t, s) \leq \frac{D s}{\varepsilon} \chi_{[\varepsilon, 2 \varepsilon]}(s) \mu_{t}(s)+D \mu_{t}(s)\left\|\psi^{t}(s)\right\| \leq D \mu_{t}(s)+D \mu_{t}(s)\left\|\psi^{t}(s)\right\|_{1} .
$$

We already saw that

$$
(t, s) \mapsto \mu_{t}(s)\left\|\psi^{t}(s)\right\|_{1} \in L^{1}\left([a, b] \times \mathbb{R}^{+}\right) .
$$

In order to conclude, we exploit (M2) to get

$$
\int_{a}^{b} \int_{0}^{\infty} \mu_{t}(s) \mathrm{d} s \mathrm{~d} t \leq \kappa(\tau) \int_{a}^{b} K_{\tau}(t) \mathrm{d} t<\infty .
$$

Hence, the DCT applies. 
V. Conclusion of the proof. Using equation (5.1), for every $t \in[a, b]$ we have

$$
\begin{aligned}
& -\frac{2}{\kappa(t)} \int_{0}^{\infty} \mu_{t}(s)\left\langle\psi(s), \partial_{t t} p\right\rangle \mathrm{d} s \\
& =\frac{2}{\kappa(t)} \int_{0}^{\infty} \mu_{t}(s)\langle\psi(s), p\rangle_{1} \mathrm{~d} s+\frac{2}{\kappa(t)}\left\|\int_{0}^{\infty} \mu_{t}(s) A^{1 / 2} \psi(s) \mathrm{d} s\right\|^{2} \\
& \quad+\frac{2}{\kappa(t)} \int_{0}^{\infty} \mu_{t}(s)\langle\psi(s), \gamma\rangle \mathrm{d} s .
\end{aligned}
$$

Then, for any $\varpi \in(0,1]$, we readily deduce from (M5) the estimate

$$
-\frac{2}{\kappa(t)} \int_{0}^{\infty} \mu_{t}(s)\left\langle\psi(s), \partial_{t t} p\right\rangle \mathrm{d} s \leq \varpi\|p\|_{1}^{2}+\frac{C}{\varpi} \kappa(t)\|\psi\|_{\mathcal{M}_{t}}^{2}+\frac{2}{\kappa(t)} \int_{0}^{\infty} \mu_{t}(s)\langle\psi(s), \gamma\rangle \mathrm{d} s .
$$

Integrating the inequality on $[a, b]$, and substituting the result into (A.8), the proof of Lemma 5.4 is finished.

\section{APPENDIX B}

\section{A Gronwall-Type Lemma in Integral Form}

Lemma B.1. Let $\tau \in \mathbb{R}$ be fixed, and let $\Lambda:[\tau, \infty) \rightarrow \mathbb{R}$ be a continuous function. Assume that for some $\varepsilon>0$ and every $b>a \geq \tau$ the integral inequality

$$
\Lambda(b)+2 \varepsilon \int_{a}^{b} \Lambda(y) \mathrm{d} y \leq \Lambda(a)+\int_{a}^{b} q_{1}(y) \Lambda(y) \mathrm{d} y+\int_{a}^{b} q_{2}(y) \mathrm{d} y
$$

holds, where $q_{1}, q_{2}$ are locally summable nonnegative functions on $[\tau, \infty)$ satisfying

$$
\int_{a}^{b} q_{1}(y) \mathrm{d} y \leq \varepsilon(b-a)+c_{1} \quad \text { and } \quad \sup _{t \geq \tau} \int_{t}^{t+1} q_{2}(y) \mathrm{d} y \leq c_{2},
$$

for some $c_{1}, c_{2} \geq 0$. Then

$$
\Lambda(t) \leq \mathrm{e}^{c_{1}}|\Lambda(\tau)| \mathrm{e}^{-\varepsilon(t-\tau)}+\frac{c_{2} \mathrm{e}^{c_{1}} \mathrm{e}^{\varepsilon}}{1-\mathrm{e}^{-\varepsilon}}
$$

for every $t \geq \tau$.

Proof. For $\nu \in(0, \varepsilon)$ arbitrarily fixed, we consider for $t \geq \tau$ the continuous positive function

having defined

$$
\Pi(t)=(|\Lambda(\tau)|+\nu) E(t, \tau)+\int_{\tau}^{t} q_{2}(y) E(t, y) \mathrm{d} y,
$$

Note that

$$
E(t, y)=\exp \left[-(2 \varepsilon-\nu)(t-y)+\int_{y}^{t} q_{1}(z) \mathrm{d} z\right]
$$

$$
E(t, y) \leq \mathrm{e}^{c_{1}} \mathrm{e}^{(\nu-\varepsilon)(t-y)}, \quad \forall t>y \geq \tau .
$$

First, we prove the inequality

$$
\Lambda(t)<\Pi(t), \quad \forall t \geq \tau .
$$

To this aim, observe that $\Lambda(\tau)<\Pi(\tau)$. Moreover, it is easily seen that for every $b>a \geq \tau$

$$
\Pi(b)+(2 \varepsilon-\nu) \int_{a}^{b} \Pi(y) \mathrm{d} y=\Pi(a)+\int_{a}^{b} q_{1}(y) \Pi(y) \mathrm{d} y+\int_{a}^{b} q_{2}(y) \mathrm{d} y .
$$


We introduce the set

$$
\mathcal{O}=\{t>\tau: \Lambda(t) \geq \Pi(t)\} .
$$

By contradiction, let $\mathcal{O}$ be nonempty. Then, defining

$$
T=\inf \mathcal{O}<\infty,
$$

due to the continuity of $\Lambda$ and $\Pi$ the following hold:

(i) $T>\tau$.

(ii) $\Lambda(T)=\Pi(T)$.

(iii) $\Lambda(t)<\Pi(t)$ for every $t \in[\tau, T)$.

In particular, for any $t \in[\tau, T)$,

$$
\Lambda(T)-\Lambda(t)>\Pi(T)-\Pi(t) .
$$

On the other hand, appealing to (B.1) and (B.4) with $a=t$ and $b=T$, we deduce

$$
\begin{aligned}
& \Lambda(T)-\Lambda(t) \leq-2 \varepsilon \int_{t}^{T} \Lambda(y) \mathrm{d} y+\int_{t}^{T} q_{1}(y) \Lambda(y) \mathrm{d} y+\int_{t}^{T} q_{2}(y) \mathrm{d} y, \\
& \Pi(T)-\Pi(t)=-(2 \varepsilon-\nu) \int_{t}^{T} \Pi(y) \mathrm{d} y+\int_{t}^{T} q_{1}(y) \Pi(y) \mathrm{d} y+\int_{t}^{T} q_{2}(y) \mathrm{d} y .
\end{aligned}
$$

Therefore, plugging these relationships into (B.5) we obtain

$$
-2 \varepsilon \int_{t}^{T} \Lambda(y) \mathrm{d} y+\int_{t}^{T} q_{1}(y) \Lambda(y) \mathrm{d} y>-(2 \varepsilon-\nu) \int_{t}^{T} \Pi(y) \mathrm{d} y+\int_{t}^{T} q_{1}(y) \Pi(y) \mathrm{d} y,
$$

and owing to (iii) we end up with

$$
-2 \varepsilon \frac{1}{T-t} \int_{t}^{T} \Lambda(y) \mathrm{d} y>-(2 \varepsilon-\nu) \frac{1}{T-t} \int_{t}^{T} \Pi(y) \mathrm{d} y
$$

At this point, since $\Lambda$ and $\Pi$ are continuous (and equal at $T$ ), we can pass to the limit as $t \rightarrow T$, so obtaining

$$
(2 \varepsilon-\nu) \Pi(T) \geq 2 \varepsilon \Lambda(T)=2 \varepsilon \Pi(T) .
$$

Since $\Pi(T)>0$, we reach the contradiction

$$
2 \varepsilon \leq 2 \varepsilon-\nu
$$

At this point, writing (B.3) explicitly, for every $t \geq \tau$ we draw

$$
\Lambda(t) \leq(|\Lambda(\tau)|+\nu) E(t, \tau)+\int_{\tau}^{t} q_{2}(y) E(t, y) \mathrm{d} y .
$$

Making use of (B.2), we get

$$
\Lambda(t) \leq \mathrm{e}^{c_{1}}(|\Lambda(\tau)|+\nu) \mathrm{e}^{(\nu-\varepsilon)(t-\tau)}+\mathrm{e}^{c_{1}} \mathrm{e}^{(\nu-\varepsilon) t} \int_{\tau}^{t} q_{2}(y) \mathrm{e}^{(\varepsilon-\nu) y} \mathrm{~d} y .
$$

Arguing analogously as in [26, Theorem 4.1], we estimate

$$
\mathrm{e}^{(\nu-\varepsilon) t} \int_{\tau}^{t} q_{2}(y) \mathrm{e}^{(\varepsilon-\nu) y} \mathrm{~d} y \leq \frac{c_{2} \mathrm{e}^{\varepsilon-\nu}}{1-\mathrm{e}^{\nu-\varepsilon}}
$$

providing

$$
\Lambda(t) \leq \mathrm{e}^{c_{1}}(|\Lambda(\tau)|+\nu) \mathrm{e}^{(\nu-\varepsilon)(t-\tau)}+\frac{c_{2} \mathrm{e}^{c_{1}} \mathrm{e}^{\varepsilon-\nu}}{1-\mathrm{e}^{\nu-\varepsilon}}
$$


As $\nu \in(0, \varepsilon)$ is arbitrary, a final limit $\nu \rightarrow 0$ gives

$$
\Lambda(t) \leq \mathrm{e}^{c_{1}}|\Lambda(\tau)| \mathrm{e}^{-\varepsilon(t-\tau)}+\frac{c_{2} \mathrm{e}^{c_{1}} \mathrm{e}^{\varepsilon}}{1-\mathrm{e}^{-\varepsilon}}
$$

as claimed.

\section{REFERENCES}

[1] A.V. Babin, M.I. Vishik, Attractors of evolution equations, North-Holland, Amsterdam, 1992.

[2] A.N. Carvalho, J.W. Cholewa, Attractors for strongly damped wave equations with critical nonlinearities, Pacific J. Math. 207 (2002), 287-310.

[3] R.M. Christensen, Theory of viscoelasticity: an introduction, Academic Press, New York, 1982.

[4] M. Conti, V. Danese, C. Giorgi, V. Pata, A model of viscoelasticity with time-dependent memory kernels, Amer. J. Math., to appear. Available at http://arxiv.org/abs/1603.07164

[5] M. Conti, V. Pata, Weakly dissipative semilinear equations of viscoelasticity, Commun. Pure Appl. Anal. 4 (2005), 705-720.

[6] M. Conti, V. Pata, Asymptotic structure of the attractor for processes on time-dependent spaces, Nonlinear Anal. Real World Appl. 19 (2014), 1-10.

[7] M. Conti, V. Pata, On the time-dependent Cattaneo law in space dimension one, Appl. Math. Comput. 259 (2015), 32-44.

[8] M. Conti, V. Pata, R. Temam, Attractors for processes on time-dependent spaces. Applications to wave equations, J. Differential Equations 255 (2013), 1254-1277.

[9] C.M. Dafermos, Asymptotic stability in viscoelasticity, Arch. Rational Mech. Anal. 37 (1970), 297308.

[10] C.M. Dafermos, Contraction semigroups and trend to equilibrium in continuum mechanics, in "Applications of Methods of Functional Analysis to Problems in Mechanics" (P. Germain and B. Nayroles, Eds.), pp.295-306, Lecture Notes in Mathematics no.503, Springer-Verlag, Berlin-New York, 1976.

[11] R. Dautray, J.-L. Lions, Mathematical analysis and numerical methods for science and technology. Vol. 5. Evolution problems. I., Springer-Verlag, Berlin, 1992.

[12] G. Duvaut, J.-L. Lions, Inequalities in mechanics and physics, Springer-Verlag, Berlin-New York, 1976.

[13] F. Dell'Oro, V. Pata, Long-term analysis of strongly damped nonlinear wave equations, Nonlinearity 24 (2011), 3413-3435.

[14] F. Di Plinio, G.S. Duane, R. Temam, Time dependent attractor for the oscillon equation, Discrete Contin. Dyn. Syst. 29 (2011), 141-167.

[15] F. Di Plinio, G.S. Duane, R. Temam, The 3-dimensional oscillon equation, Boll. Unione Mat. Ital. 5 (2012), 19-53.

[16] A.D. Drozdov, V.B. Kolmanovskii, Stability in viscoelasticity, North-Holland, Amsterdam, 1994.

[17] A. Eden, V. Kalantarov, Finite dimensional attractors for a class of semilinear wave equations, Turkish J. Math. 20 (1996), 425-450.

[18] M. Fabrizio, A. Morro, Mathematical problems in linear viscoelasticity, SIAM Studies Appl. Math. 12, Philadelphia, PA, 1992.

[19] S. Gatti, A. Miranville, V. Pata, S. Zelik, Attractors for semi-linear equations of viscoelasticity with very low dissipation, Rocky Mountain J. Math. 38 (2008), 1117-1138.

[20] J.M. Ghidaglia, A. Marzocchi, Longtime behaviour of strongly damped wave equations, global attractors and their dimension, SIAM J. Math. Anal. 22 (1991), 879-895.

[21] M. Grasselli, V. Pata, Uniform attractors of nonautonomous systems with memory, in "Evolution Equations, Semigroups and Functional Analysis" (A. Lorenzi and B. Ruf, Eds.), pp.155-178, Progr. Nonlinear Differential Equations Appl. no.50, Birkhäuser, Boston, 2002.

[22] J.K. Hale, Asymptotic behavior of dissipative systems, Amer. Math. Soc., Providence, 1988.

[23] A. Haraux, Systèmes dynamiques dissipatifs et applications, Masson, Paris, 1991.

[24] P. Massat, Limiting behavior for strongly damped nonlinear wave equations, J. Differential Equations 48 (1983), 334-349.

[25] V. Pata, Exponential stability in linear viscoelasticity, Quart. Appl. Math. 64 (2006), 499-513. 
[26] V. Pata, G. Prouse, M.I. Vishik, Traveling waves of dissipative non-autonomous hyperbolic equations in a strip, Adv. Differential Equations 3 (1998), 249-270.

[27] V. Pata, M. Squassina, On the strongly damped wave equation, Comm. Math. Phys. 253 (2005), $511-533$.

[28] V. Pata and S. Zelik, Smooth attractors for strongly damped wave equations, Nonlinearity 19 (2006), $1495-1506$.

[29] V. Pata, A. Zucchi, Attractors for a damped hyperbolic equation with linear memory, Adv. Math. Sci. Appl. 11 (2001), 505-529.

[30] M. Renardy, W.J. Hrusa, J.A. Nohel, Mathematical problems in viscoelasticity, Longman Scientific \& Technical, Harlow John Wiley \& Sons, Inc., New York, 1987.

[31] J. Simon, Compact sets in the space $L^{p}(0, T ; B)$, Annali Mat. Pura Appl. 146 (1987), 65-96.

[32] R. Temam, Infinite-dimensional dynamical systems in mechanics and physics, Springer, New York, 1997.

[33] G.F. Webb, Existence and asymptotic behavior for a strongly damped nonlinear wave equation, Canad. J. Math. 32 (1980), 631-643.

Politecnico di Milano - Dipartimento di Matematica

Via Bonardi 9, 20133 Milano, Italy

E-mail address: monica.conti@polimi.it (M. Conti)

E-mail address: valeria.danese@polimi.it (V. Danese)

E-mail address: vittorino.pata@polimi.it (V. Pata) 